\title{
Synthesis of novel palladium allyl complexes bearing heteroditopic NHC-S ligands. Kinetic study on the carbene exchange between bis-carbene palladium allyl complexes
}

\author{
Luciano Canovese $^{\mathrm{a}, *}$, Fabiano Visentin ${ }^{\mathrm{a}}$, Carlo Levi ${ }^{\mathrm{a}}$, Claudio Santo ${ }^{\mathrm{a}}$, Valerio Bertolasi ${ }^{\mathrm{b}}$ \\ a Dipartimento di Scienze Molecolari e Nanosistemi, Università Ca' Foscari, Calle Larga S. Marta, 30129 Venice, Italy \\ ${ }^{\mathrm{b}}$ Dipartimento di Chimica e Centro di Strutturistica Difrattometrica, Università di Ferrara, Italy
}

\section{A R T I C L E I N F O}

\section{Article history:}

Received 22 October 2012

Received in revised form

15 January 2013

Accepted 18 January 2013

\section{Keywords:}

Palladium allyl complexes

Heteroditopic carbene ligands

Carbene exchange reaction

\begin{abstract}
A B S T R A C T
We have synthesized several novel palladium allyl and 1,1-dimethylallyl complexes bearing different heteroditopic NHC-S ligands giving rise to a five-membered chelate ring with the metal center. We were able to synthesize some homoleptic bis-carbene allyl derivatives by taking advantage of the hemilability of the thioetheric sulfur. Attempts at preparing mixed bis-carbene complexes bearing two different heteroditopic carbenes (i.e. NHC-S and NHC-Py) simultaneously coordinated to the palladium center lead to a carbene transmetalation with the formation of a statistically distributed equilibrium mixture of the two pure homoleptic and of the mixed bis-carbene palladium allyl complexes in solution. In two different cases the rate of the equilibrium reaction was measured and a mechanistic hypothesis provided. Finally, we have determined the solid state structures of a complex bearing only one NHC-S heteroditopic carbene and of the bis-carbene (NHC-S, NHC-Py) palladium allyl derivatives.
\end{abstract}

(c) 2013 Elsevier B.V. All rights reserved.

\section{Introduction}

The family of efficient $\sigma$ donor $\mathrm{N}$-heterocyclic carbenes (NHC) which impart a remarkable stability and catalytic performances to their transition metal derivatives [1], has been soon extended to include a new class of bidentate ligands carrying another coordinating functionality. The coordination of heteroditopic NHC-E $(\mathrm{E}=\mathrm{P}, \mathrm{N}, \mathrm{O})$ ligands to transition metals yields an important class of new compounds [2]. In particular, the complexes characterized by a secondary, labile heteroatom act as efficient and stable catalysts since the dangling wing can restore the starting complexes by re-coordination of the site made vacant by their catalytic activity [3]. We have been long involved in the study of the synthesis, behavior in solution and reactivity of $\operatorname{Pd}(0)$ and $\operatorname{Pd}(\mathrm{II})$ complexes with bidentate or terdentate ligands bearing at least one thioether function [4]. Thus, owing to the not particularly high number of heteroditopic bidentate sulfur-carbene ligands in the literature [5], we decided to synthesize some new $\mathrm{NHC}-\mathrm{S}$ chelating moieties and the corresponding palladium allyl complexes. We undertook such an investigation since the majority of NHC palladium allyl complexes displaying a marked catalytic activity [6] is mainly stabilized

\footnotetext{
* Corresponding author. Tel.: +39 (0)41 2348571; fax: +39 (0)41 2348517.

E-mail address: cano@unive.it (L. Canovese).
}

by monodentate carbene ligands [7]. On the contrary, the bidentate heteroditopic derivatives are comparatively less common [8] whereas the NHC-S complexes represent a rarity [5c,d], although the thioether wing might impart peculiar catalytic properties to their complexes owing to the stereogenic nature of the coordinated sulfur [5k].

The NHC-S ligands that can form five-membered $\mathrm{C}-\mathrm{S}$ ring upon coordination to a metal center, are also rare $[5 \mathrm{~h}, \mathrm{l}, \mathrm{n}]$ and no allyl derivatives with this kind of spectator ligands are reported in the literature. In addition, it is known that two different heteroditopic carbene ligands bearing nitrogen or sulfur as secondary atom can simultaneously coordinate to either $\operatorname{Pd}(0)[5 n, 9]$ or $\operatorname{Pd}($ II) [10] and this very fact might suggest interesting studies on the possible carbene exchange. As a matter of fact, despite the great deal of papers on the transmetalation reactions involving different metals such as the carbonyl carbene complexes of the group 6 metals [11] or $\operatorname{AgBr}\left(\mathrm{NR}, \mathrm{NCH}_{2} \mathrm{R}^{\prime}-\mathrm{NHC}\right)$ substrates [12] with several different transition metal derivatives, to the best of our knowledge no studies on the carbene exchange between Pd(II) compounds have appeared in the literature, although Caddick and Cloke have already demonstrated the feasibility of carbene exchange in $\operatorname{Pd}(0)$ complexes, whereas Yamamoto and Espinet have found that the aryl groups can exchange between $\mathrm{Pd}(\mathrm{II})$ aryl complexes [13]. In the present study we show that the allyl palladium derivatives bearing five-membered NHC-S ligands can be easily synthesized and that 
the transmetalation reaction involving exchange of the heterobidentate ligands between different palladium allyl complexes takes place through an associative mechanism that is strongly dependent on the nature of the dangling wing.

\section{Results and discussion}

\subsection{Palladium allyl complexes}

We have firstly synthesized the imidazolium salts described in Scheme 1 by reacting the suitable R-imidazole with chloromethylmethyl sulfide, chloromethyl-phenyl sulfide or chloromethylpyridine in acetonitrile in the presence of $\mathrm{KBr}$ (Scheme 1):

$\mathbf{1 a}$ and $\mathbf{1 c}$ represent newly synthesized ligands, whereas $\mathbf{1 b}$ [5l], 1d [ $5 n$ ] are literature compounds. All the bidentate NHC-S ligands in Scheme 1 form a five-membered chelate ring upon complexation with the group 10 metals.

The silver carbene derivatives (2) were then obtained in reasonable yield (70-90\%) by reacting the imidazolium salts 1 with $\mathrm{Ag}_{2} \mathrm{O}$ in $\mathrm{CH}_{2} \mathrm{Cl}_{2}$ [2f,g]. Moreover, for reasons that will be discussed further on and according to published procedures we have synthesized the complex \{[1-(2-pyridyl)methylene-3-methyl]imidazolyl-2-ene\}silver bromide (2e) [14]. The disappearance of the broad signal of the acidic $\mathrm{C}_{2}-\mathrm{H}$ proton of the imidazolium salt at $\sim 10 \mathrm{ppm}$ in the ${ }^{1} \mathrm{H}$ NMR spectrum and the appearance of the signal related to the coordinated carbene carbon at $\sim 180 \mathrm{ppm}$ in the ${ }^{13} \mathrm{C}$ NMR testified the progress of the reaction. Finally, taking advantage of the synthetic strategy based on $\mathrm{Ag}(\mathrm{I})$ carbene transfer developed by Lin et al. [15] and widely employed by other authors, we have carried out the synthesis of the title complexes 4 by means of a slight modification of Li's method [16]. Thus, the transfer of carbene from the $\mathrm{Ag}(\mathrm{I})$ derivative to the palladium allyl chloro dimer was followed by dechlorination of the firstly formed monodentate carbene allyl chloro complexes 3 with a solution of $\mathrm{NaClO}_{4}$ in methanol (Scheme 2):

The [1-(2-pyridyl)methylene-3-methyl]imidazolyl-2-ene derivatives $\mathbf{2 e}$ [14], 3e and $\mathbf{4 e}$ obtained by similar procedure, are shown in Chart 1.

The structure of the chelate allyl complexes $\mathbf{4 a}-\mathbf{d}$ in solution is clearly apparent from comparison of their NMR spectra with those of the derivatives 3 (vide post). The heterotopicity of spectator ligands is reflected in the spectroscopic behavior of the allyl fragment for which five distinct signals are detectable in the ${ }^{1} \mathrm{H}$ NMR spectra at RT. In particular, the syn and anti peripheral allyl protons trans to carbene resonate at higher field than the corresponding protons trans to the thioether group (Supplementary material; Fig. 1SM). In addition, the coordination of the sulfur is inferred from the diastereotopism of the endocyclic $\mathrm{CH}_{2}-\mathrm{S}$ protons resonating as an $\mathrm{AB}$ system centered at ca. $5.5 \mathrm{ppm}$ [17]. The allyl palladium complexes bearing the thioetheric sulfur as secondary

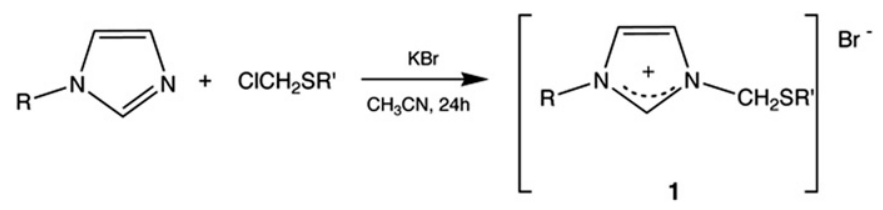

$\mathrm{R}^{\prime}=\mathrm{Me}$

$\mathrm{R}=\mathrm{Me}$ (1a); Mesityl (1b); di-i-Propylphenyl (1c)

$\mathrm{R}^{\prime}=\mathrm{Ph}$

$\mathrm{R}=\mathrm{Me}$ (1d); Mesityl (1e); di-i-Propylphenyl (1f) donating atom often undergo rapid inversion of the sulfur absolute configuration at RT. At low temperature such an inversion is frozen and the consequent formation of a second chiral center becomes apparent since a couple of diastereoisomers due to the different mutual orientation between the allyl central atom and the substituent at the chiral sulfur can be detected [4]. As a matter of fact, the low temperature ${ }^{1} \mathrm{H}$ NMR spectra of the title complexes ( $\leq 193 \mathrm{~K}$ ) display the splitting of each group of signals related to the allyl protons into two further groups of signals with different intensity, which can be traced back to the different diastereoisomers (Supplementary material; Fig. 2 SM).

The RT ${ }^{13} \mathrm{C}$ NMR spectra display two well separated signals due to the peripheral allyl carbons. The allyl carbon trans to carbene affected by the highest trans influence [18] resonates at ca. 70 ppm, whereas the allyl carbon trans to sulfur is detected at ca. $50 \mathrm{ppm}$. Finally, the coordinated carbon of the carbene moiety resonates at ca. 180 ppm.

Moreover, the nature of the cationic complexes $\mathbf{4}$ is confirmed by their IR spectra displaying the characteristic stretching and bending bands of the $\mathrm{ClO}_{4}{ }^{-}$counter-ion at ca. 1080 and $620 \mathrm{~cm}^{-1}$, respectively.

When $\mathrm{NaClO}_{4}$ is not added, the reaction of the complexes of type 2 with palladium chloro allyl dimer yields the corresponding monodentate NHC chloro allyl derivatives $\mathbf{3}$ (Scheme 2). Complexes 3a-d have not been isolated but their ${ }^{1} \mathrm{H}$ NMR spectra have been recorded in situ. They are characterized by a remarkable up-field shift of both the syn and anti allyl protons trans to chloride with respect to those trans to sulfur in complexes $\mathbf{4 a}-\mathbf{d}$, whereas those trans to the carbene carbon do not shift significantly [7a,19]. At variance with chloride, which confirms its remarkable coordinative capability in non coordinating solvents [16], this phenomenon is strictly related to the not particularly strong coordinative nature of the thioetheric sulfur toward Pd(II) [4] which in this case is almost independent of the substituent at sulfur. The $\mathrm{CH}_{2}-\mathrm{S}$ protons of complexes $\mathbf{3 a}-\mathbf{d}$ are detected as an $\mathrm{AB}$ system which is however considerably narrower than that of the corresponding derivatives $\mathbf{4 a}-\mathbf{d}$. Such a not unprecedented phenomenon is probably due to sterically hampered free rotation of the substituents at the uncoordinated sulfur [20].

\subsection{Palladium $\mathrm{Me}_{2}$-allyl complexes}

Similarly to the synthetic protocol reported in Scheme 2, the transmetalation reaction between the silver derivatives $\mathbf{2}$ and the 1,1-dimethyl allyl palladium chloro dimer in the presence of $\mathrm{NaClO}_{4}$ yields the complexes $\mathbf{5}$ (Chart 2). Significantly, at RT only the isomer with the di-methyl substituted allyl termini trans to the carbene is present in solution.

The assignment of the structure of complexes $\mathbf{5}$ in solution is easily achieved by HMBC analysis which displays an intense crosspeak between the carbene carbon and the protons of the methyl substituents of the terminal allyl carbon (Supplementary material; Fig. $3 \mathrm{SM}$ ). As a matter of fact, it was shown that such phenomenon is observable when the carbene and the bis-substituted allyl carbon occupy a mutual trans position [21]. The hypothesized structure was definitely confirmed by X-ray crystallographic analysis of $\mathbf{5 b}$ (vide infra).

The marked trans influence and the consequent trans effect exerted by unhindered carbenes when compared with pyridine and even with phosphines was clearly pointed out by Danopoulos and co-workers in the case of the protonation of dimethyl palladium complexes bearing unsymmetrical phosphino-pyridine spectator ligands [8a]. Therefore, it is not quite surprising that the trans influence exerted by the carbene forces the less coordinating allyl termini to the trans position. However, the complete selectivity of 


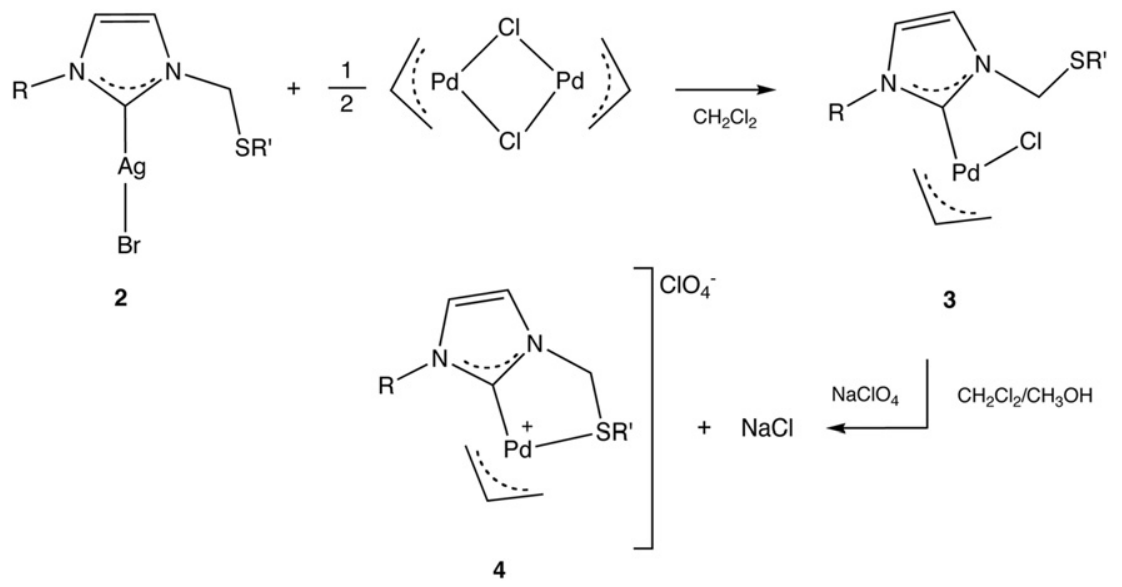

$$
\begin{aligned}
& R^{\prime}=M e \\
& R=M e(2 a, 3 a, 4 a) ; \text { Mesityl }(2 b, 3 b, 4 b) ; \text { di-i-Propylphenyl }(2 c, 3 c, 4 c) \\
& R^{\prime}=P h \\
& R=M e(2 d, 3 d, 4 d)
\end{aligned}
$$

Scheme 2.

the phenomenon observed in the case of all palladium $\mathrm{Me}_{2}$-allyl complexes $\mathbf{5}$ is remarkable since it was never observed in similar complexes bearing different heteroditopic ligands [22].

\subsection{Palladium bis-carbene allyl complexes}

According to Scheme 3, addition of the $\mathrm{Ag}(\mathrm{I})$ carbene derivatives 2 to complexes $\mathbf{4}$ causes fast and complete formation of the biscarbene allyl palladium substrates 6 [23]. It is noteworthy that these reactions are effective only when at least one of the imidazole substituents is the poorly hindered methyl group.

Owing to the enhanced symmetry of complexes $\mathbf{6}$, the ensuing RT ${ }^{1} \mathrm{H}$ spectra appear considerably simplified with respect to those of complexes 4. Thus, only one doublet ascribable to the syn and one for the anti protons together with the multiplet of the central allyl proton integrating 2:2:1, respectively, are detectable. Moreover, the protons of the $\mathrm{CH}_{3}-\mathrm{N}$ group coordinated to both ligands resonate as an independent singlet. Similar behavior is observed in the case of the $\mathrm{CH}_{2}-\mathrm{S}$ protons although they are no longer diastereotopic as a consequence of the de-coordination of sulfur. Consistently, in the ${ }^{13} \mathrm{C}$ NMR spectra both carbons of the allyl termini resonate as only one singlet. Finally, the carbene carbons resonate at ca. $180 \mathrm{ppm}$ without any significant shift with respect to those of the starting complexes 4.

Following the same synthetic protocol used for the synthesis of complexes $\mathbf{6 a}, \mathbf{b}$ (vide supra), we synthesized the complex $\mathbf{6 e}$
(Chart 3) by reacting the complex $2 \mathbf{e}$ with $4 \mathbf{4 e}$. Complex $6 \mathbf{e}$ behaves in solution similarly to derivatives $\mathbf{6 a}$ and $\mathbf{6 b}$ and therefore the relevant features of their ${ }^{1} \mathrm{H}$ and ${ }^{13} \mathrm{C}$ NMR spectra are quite comparable (see Experimental).

In order to rationalize the reaction rates observed for the transmetalation between the complexes $\mathbf{6 a}$ and $\mathbf{6 e}$ and to obtain an unambiguous kinetic response, we have synthesized the monodentate ligands 1-methyl-3-(benzyl)-2,3-dihydro- $1 H$-imidazolium bromide 1f and 1-(4-methyl-benzyl)-3-methyl-2,3-dihydro- $1 \mathrm{H}$-imidazolium bromide $1 \mathrm{~g}$ by quaternization of the 1 -methyl- $1 \mathrm{H}$-imidazole with 1 chloromethyl-benzene or 1-chloromethyl-4-methyl-benzene, respectively in acetonitrile in the presence of $\mathrm{KBr}$. The corresponding bis-carbene allyl derivatives were prepared by dechlorination with $\mathrm{AgBF}_{4}$ of the complexes $\mathbf{3 f}$ and $\mathbf{3 g}$ which were obtained by reacting the substrates $\mathbf{2 f}$ and $\mathbf{2 g}$ with $\left[\mathrm{Pd}\left(\eta^{3}-\text { allyl }\right)(\mu-\mathrm{Cl})\right]_{2}$ followed by a further attack of $\mathbf{2 f}$ or $\mathbf{2 g}$ at the solvato species (Scheme 4). It is noteworthy that similar complexes were only identified by Cavell and coworkers as by-products in the synthesis of mixed NHC-phosphine Pd allyl derivatives, but never isolated [24].

\subsection{Exchange between carbenes in Pd(II) allyl complexes}

The carbene transfer between $\operatorname{Pd}(0)$ complexes has been described by Caddick and Cloke [13]. However, a similar reaction involving Pd(II) carbene derivatives was never studied. Such an exchange reaction was even ruled out by Chen and co-workers on<smiles></smiles>

$2 e$<smiles></smiles>

$3 e$<smiles></smiles>

$4 e$

Chart 1. Complexes $2 \mathbf{e}, 3 \mathbf{e}$ and $4 \mathbf{e}$. 


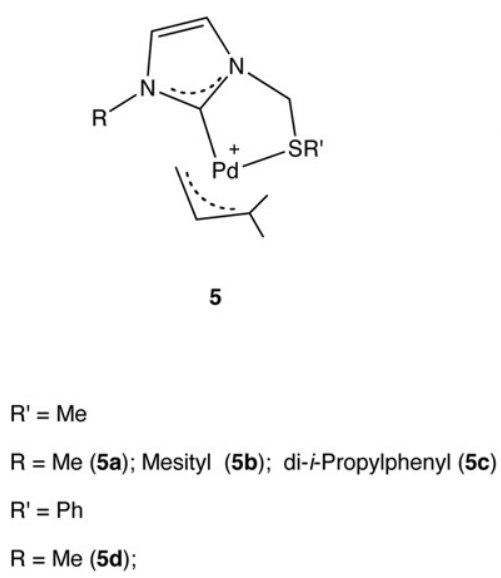

Chart 2. Complexes 5

the basis of the observation that dimeric complexes of the type $\left[\mathrm{Pd}(\mathrm{NHC}) \mathrm{Cl}_{2}\right]_{2}$ never convert into the $\left[\mathrm{Pd}(\mathrm{NHC})_{2} \mathrm{Cl}_{2}\right.$ ] species [25]. However, in an attempt at preparing mixed carbene derivatives in situ we have reacted equimolecular solutions of the complexes $4 \mathbf{a}$ and $2 \mathbf{e}$ in $\mathrm{CD}_{2} \mathrm{Cl}_{2}$ at $\mathrm{RT}$ in an NMR test tube. It was clear that the immediately formed complex 7 slowly evolves into an equilibrium mixture of three different statistically distributed $(50,25,25 \%$, respectively) chemical species, namely complexes $7, \mathbf{6 a}$ and $\mathbf{6 e}$ (Scheme 5).

We therefore surmise that the transmetalation involving two palladium(II) complexes is possible in some cases. Therefore, with the aim of exploring the feasibility and the potentiality of such reaction, we decided to plan further experiments in which equimolecular solutions of two homoleptic but different bis-carbene allyl palladium complexes were mixed together. Preliminary ${ }^{1} \mathrm{H}$ NMR experiments involving complexes $\mathbf{6 a}, \mathbf{6 b}$ and $\mathbf{6 e}$ clearly indicated that the reaction occurs, although not completely, and again an equilibrium mixture was detected at the end of the process. As expected, owing to the presumably very similar $\Delta G_{f}^{\circ}$ of all the complexes involved, the products of the equilibrium reaction were obtained in a statistical distribution $\left(K_{\mathrm{E}} \cong 4\right)$ [22c]. Therefore, we decided to study quantitatively by ${ }^{1} \mathrm{H}$ NMR the reactions involving complexes 6a, $6 \mathbf{e}$ and $6 \mathbf{6 a}, 6 \mathbf{f}$ and eventually $\mathbf{6 f}, \mathbf{6 g}$. Thus, we have set up kinetic experiments in an attempt at determining the mechanism of the transmetalation reaction. In Scheme 6, the reactions studied and the related reaction products $\mathbf{7 , 8}$ and $\mathbf{9}$, are listed.

Table 1 reports a summary of the kinetic data determined by non linear regression analysis of the concentration-time profiles as deduced from the NMR integration of selected signals of the species

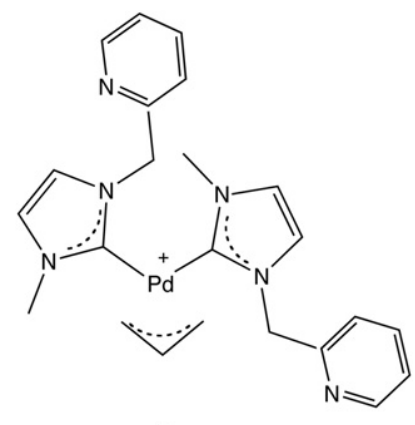

$6 e$

Chart 3. Complex 6 e

involved. In Fig. 1 we show the ${ }^{1} \mathrm{H}$ NMR spectra and the concentration $v$ s. time plot in the case of the reaction between $6 \mathbf{a}$ and $\mathbf{6 f}$ complexes. The mathematical treatment was carried out by a locally adapted program written in the SCIENTIST ${ }^{\circledR}$ environment. All the concentration $v s$. time plots and the mathematical analysis are reported in Figs. 4 SM, 5 MI, 6 SM and equation (1) SM in Supplementary material.

Although the equilibrium constants $K_{\mathrm{E}}$ calculated as the ratios of rate constants reasonably fit the estimated values obtained from integration of the ${ }^{1} \mathrm{H}$ NMR signals of the equilibrium mixture, the reaction rates raise some perplexity since the observed trend is apparently inconsistent.

As a matter of fact, the rate of reaction between the complexes bearing a coordinating function in the dangling wing (6a and $\mathbf{6 e}$ to give 7 ) is higher than that related to the formation of $\mathbf{8}$ (only $\mathbf{6 a}$ bears a coordinating function) but smaller than that for 7 (no coordinating function is present in the wings of $\mathbf{6 f}$ and $\mathbf{6 g}$ ). We think that a possible interpretation shouldn't take into account the activation energy required for the formation of the species that reasonably represents the intermediates involved in different reactions. Thus, in all cases studied the carbene transfer occurs as a consequence of the formation of a dimeric transition state which can be represented in Chart 4 in the case of complexes 7, 8 and $\mathbf{9}$, according to the classical theory of nucleophilic attack in $\mathrm{d}^{8}$ complexes:

Therefore, the formation of $\mathbf{9}$ involves an intermediate of type $\mathbf{I}_{\mathbf{1}}$ whereas the presence of a second coordinating function in the dangling wing might impose the formation of other types of intermediates ( $\mathbf{I}_{2}$ and $\mathbf{I}_{3}$ ) which would collapse slowly into the products $\mathbf{7}$ and $\mathbf{8}$. The doubly bridged intermediate $\mathbf{I}_{\mathbf{2}}$ appears to be more reactive than $\mathbf{I}_{\mathbf{3}}$ probably because its more compact structure favors the subsequent interaction between the carbene carbons and the metal.

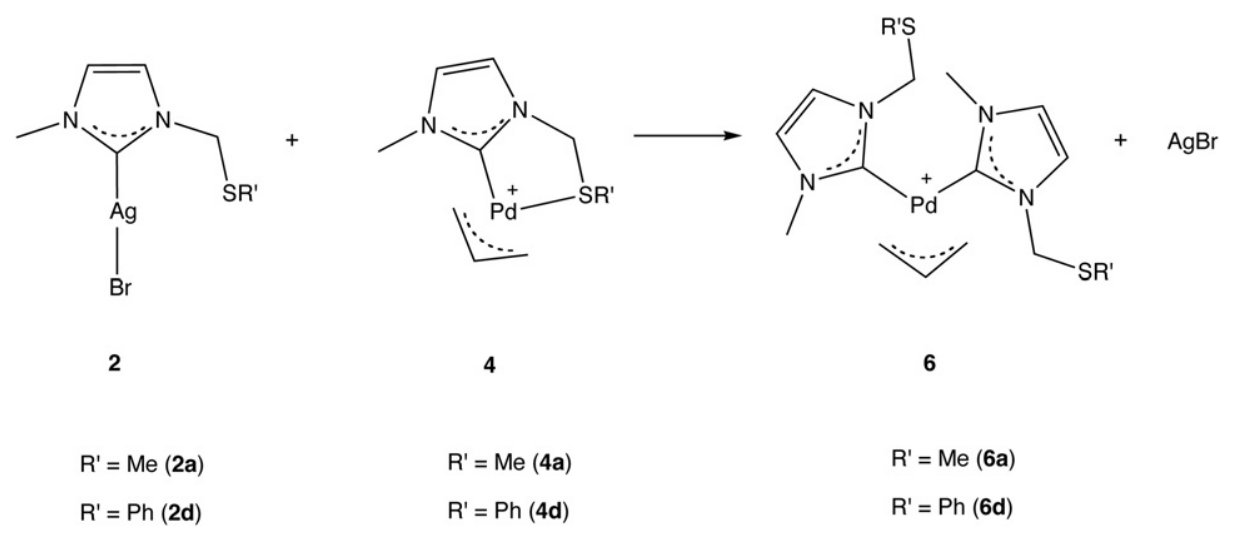

Scheme 3. 
<smiles></smiles>

2<smiles></smiles>

3<smiles>C=C[Te][Te]Cl</smiles>

$\overrightarrow{\mathrm{CH}_{2} \mathrm{Cl}_{2}}$<smiles>CCC</smiles><smiles>CC(C)[18F]</smiles>

2<smiles></smiles>

3

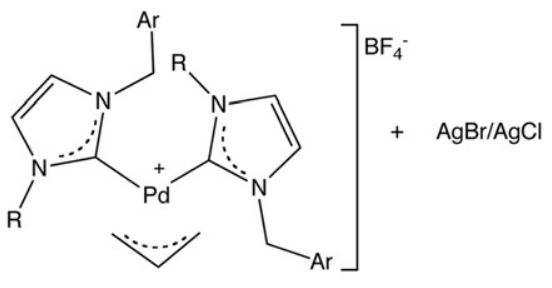

6

$$
\begin{aligned}
& \mathrm{Ar}=\mathrm{C}_{6} \mathrm{H}_{5}(\mathbf{2 h}, 3 \mathbf{h}, 6 \mathbf{h}) \\
& \mathrm{Ar}=\mathrm{C}_{6} \mathrm{H}_{5}-\mathrm{Me}-4(2 \mathbf{i}, 3 \mathbf{i}, 6 \mathbf{i})
\end{aligned}
$$

Scheme 4 .

\subsection{Crystal structure determinations}

The asymmetric unit of compound $\mathbf{5 b}$ contains two independent ionic couples. ORTEP [26] views of both independent Pd(II) cationic complexes 5b and of the $\mathrm{Pd}(\mathrm{II})$ cationic complex $\mathbf{6 d}$ are reported in Figs. 2 and 3. A selection of bond distances and angles is given in Tables 2 and 3. In complex $\mathbf{5 b}$ the palladium is bonded to a dissymmetric NHC bidentate ligand through the carbene carbon and the sulfur atom of the methylthiomethyl N-substituent and $\eta^{3}$-coordinated to the 1,1-dimethyl substituted allyl group. The trans effect exerted by the carbene atom can be evidenced by the longer Pd1-C3 distances of 2.235(3) $\AA$ as compared to those where a terminal allyl carbon is in trans position to the sulfur atoms: $(\mathrm{Pd} 1-\mathrm{C} 1=2.108(4)$ and $2.131(4) \AA$ for the cations $\mathrm{A}$ and $\mathrm{B}$, respectively).

In the cationic complex $\mathbf{6 d}$ the palladium is bonded to two carbene carbons of two dissymmetric NHC ligands and $\eta^{3}$-coordinated to the allyl group.

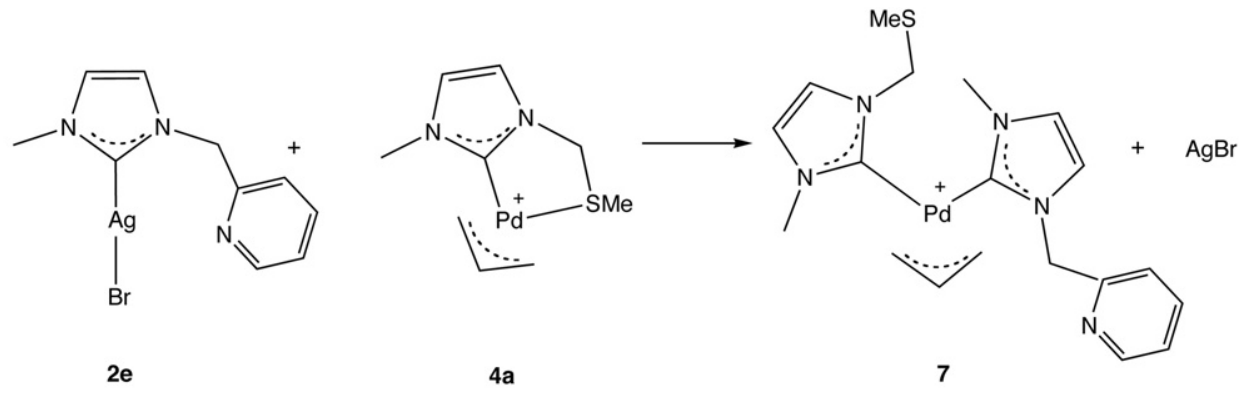<smiles></smiles>

Scheme 5. 


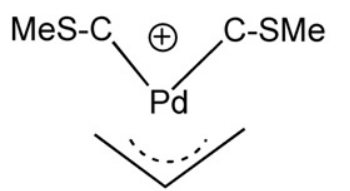

6a; $25 \%$

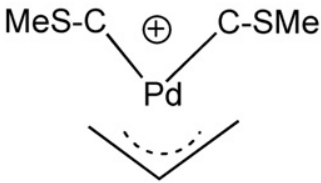

6a; $25 \%$

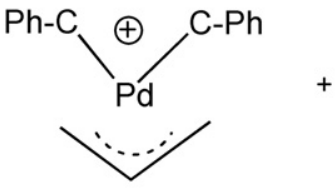

6h; $25 \%$<smiles>C/C=C\[Pb]C[18O]</smiles>

6g; $25 \%$

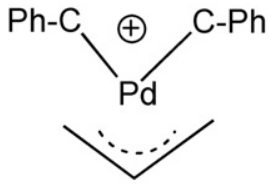

6h; $25 \%$

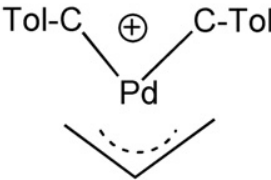

6i, $25 \%$

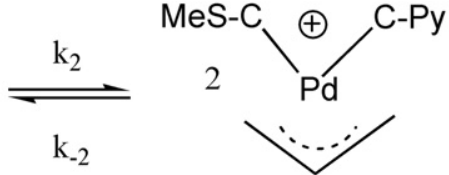

$7 ; 50 \%$

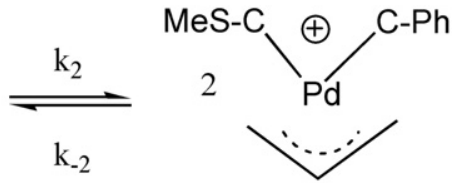

$8 ; 50 \%$

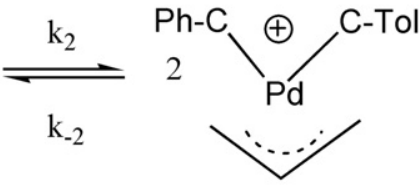

9, $50 \%$

Scheme 6.

The 1,1-dimethyl substituted allyl group is almost perpendicular to the $\mathrm{Pd}(\mathrm{II})$ basal coordination plane in both cationic complexes of compound $\mathbf{5 b}$, forming dihedral angles in the range of 85.2(6)$86.9(2)^{\circ}$ (Table 2 ), whereas in the cationic complex 6d the allyl group forms an angle of $68.2(7)^{\circ}$ with the basal $\mathrm{Pd}(\mathrm{II})$ coordination plane (Table 3).

\section{Conclusions}

We have synthesized and characterized some potentially fivemembered NHC-S ligands and the corresponding new chelate palladium allyl derivatives by transmetalation between the silver carbene derivatives $\mathrm{AgBr}\left(\mathrm{NR}, \mathrm{NCH}_{2} \mathrm{SR}^{\prime}-\mathrm{NHC}\right)$ and the $\mathrm{Pd}(\mathrm{II})$ allyl chloro dimer, followed by dechlorination of the monodentate derivative with $\mathrm{NaClO}_{4}$. In the case of the reaction involving the dimer $\left[\mathrm{Pd}\left(\eta^{2}-\mathrm{Me}_{2} \text {-ally }\right)(\mu-\mathrm{Cl})\right]_{2}$ only the complex with the bis-substituted allyl terminus trans to the carbene carbon was obtained in any case. Furthermore, we have synthesized and characterized some palladium bis-carbene allyl derivatives and shown that the carbene fragments can be easily exchanged between different bis-carbene complexes giving rise to an equilibrium situation in which the starting and final complexes generate a statistically distributed

\section{Table 1}

Second order rate constants and calculated and estimated equilibrium constants for the carbene exchange reaction in complexes $6 \mathbf{6 a}, 6 \mathbf{6} ; \mathbf{6 a}, \mathbf{6 f} ; \mathbf{6 f}, \mathbf{6 g}$.

\begin{tabular}{llll}
\hline $\begin{array}{l}\text { Formed } \\
\text { complex }\end{array}$ & $\begin{array}{l}k_{2} \times 10^{-2} \\
\left(\mathrm{~mol}^{-1} \mathrm{dm}^{3} \mathrm{~s}^{-1}\right)\end{array}$ & $\begin{array}{l}k_{-2} \times 10^{-2} \\
\left(\mathrm{~mol}^{-1} \mathrm{dm}^{3} \mathrm{~s}^{-1}\right)\end{array}$ & $K_{\mathrm{E}}$ \\
\hline $\mathbf{7}$ & $4.9 \pm 0.2$ & $1.2 \pm 0.2$ & $4.1 \pm 0.7^{\mathrm{a}}\left(\sim 4^{\mathrm{b}}\right)$ \\
$\mathbf{8}$ & $0.22 \pm 0.01$ & $0.067 \pm 0.001$ & $3.2 \pm 0.2^{\mathrm{a}}\left(\sim 4^{\mathrm{b}}\right)$ \\
$\mathbf{9}$ & Fast & Fast & $\left(\sim 4^{\mathrm{b}}\right)$ \\
\hline
\end{tabular}

a Calculated values $\left(K_{\mathrm{E}}=k_{2} / k_{-2}\right)$.

b Estimated value obtained from ${ }^{1} \mathrm{H}$ NMR integration of the equilibrium mixture. equilibrium mixture. When possible, we have calculated the reaction rates relating to the equilibrium reaction and surmised a plausible mechanism.

\section{Experimental}

\subsection{Solvents and reagents}

All solvents were purified by standard procedures and distilled under argon immediately before use. 1D- and 2D-NMR spectra were recorded using a Bruker 300 Avance spectrometer. Chemical shifts (ppm) are given related to TMS $\left({ }^{1} \mathrm{H}\right.$ and ${ }^{13} \mathrm{C}$ NMR). Peaks are

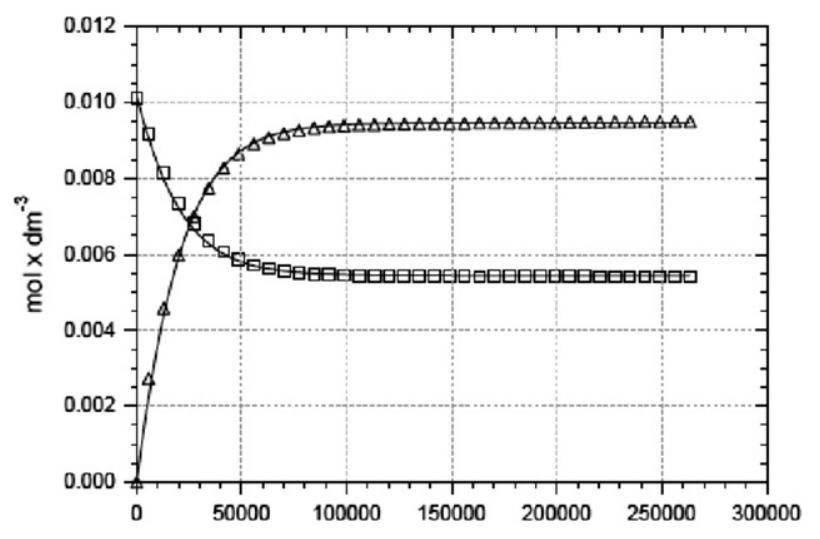

$\mathrm{t}(\mathbf{s})$

Fig. 1. Simplified plot of concentration $v$. time (s) for the reaction: $\mathbf{6 a}+\mathbf{6 e}=2 \cdot \mathbf{8}$ $[\mathbf{6 a}]=9.7 \times 10^{-3},[\mathbf{6 e}]=1.3 \times 10^{-2}\left(\mathrm{~mol} \mathrm{dm}^{-3}\right)$. For the sake of clarity the concentration of $\mathbf{6 e}$ and several concentrations of $\mathbf{6 a}(\square)$ and $\mathbf{8}(\boldsymbol{\Delta})$ vs time data (three every four) were omitted. 


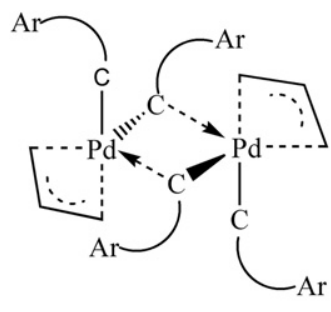

$I_{1}: 6 f+6 g=9$

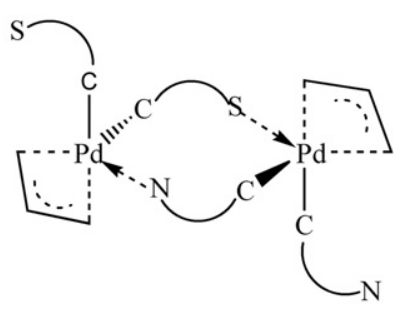

$I_{2}: 6 a+6 e=7$

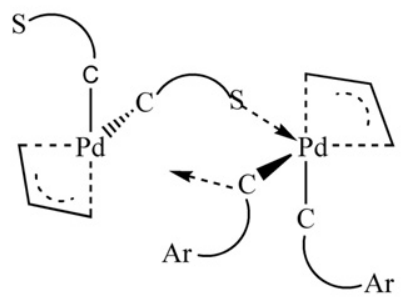

$I_{3}: 6 a+6 f=8$

Chart 4. Dimeric transition state formed by the complexes 7, 8 and $\mathbf{9 .}$

labeled as singlet $(\mathrm{s})$, doublet $(\mathrm{d})$, triplet $(\mathrm{t})$, quartet $(\mathrm{q})$, multiplet $(\mathrm{m})$ and broad $(\mathrm{b})$. The proton and carbon assignments were carried out by ${ }^{1} \mathrm{H}-2 \mathrm{D}$ COSY, ${ }^{1} \mathrm{H}-2 \mathrm{D}$ NOESY, ${ }^{1} \mathrm{H}-{ }^{13} \mathrm{C}$ HMQC and HMBC experiments.

IR spectra were recorded on a Perkin-Elmer Spectrum One spectrophotometer.

\subsection{Kinetic measurements}

The transmetalation reactions were followed by ${ }^{1} \mathrm{H}$ NMR technique by dissolving one of the complexes under study (6a or $\mathbf{6 f}$ ) in $0.8 \mathrm{~mL}$ of $\mathrm{CD}_{2} \mathrm{Cl}_{2}$ ([complex] $\sim 0.01 \mathrm{~mol} \mathrm{dm}^{-3}$ ) at $25^{\circ} \mathrm{C}$. An equimolar aliquot of the reacting counterpart $(\mathbf{6 e}, \mathbf{6 f}$ or $\mathbf{6 g}$ ) was added as a solid and the reaction was followed to completion by monitoring the disappearance of the starting complexes and the concomitant appearance of the final bis-carbene species. The plots of the concentration-time profiles of the substrates involved (calculated from integration of the ${ }^{1} \mathrm{H}$ NMR relevant peaks) are reported

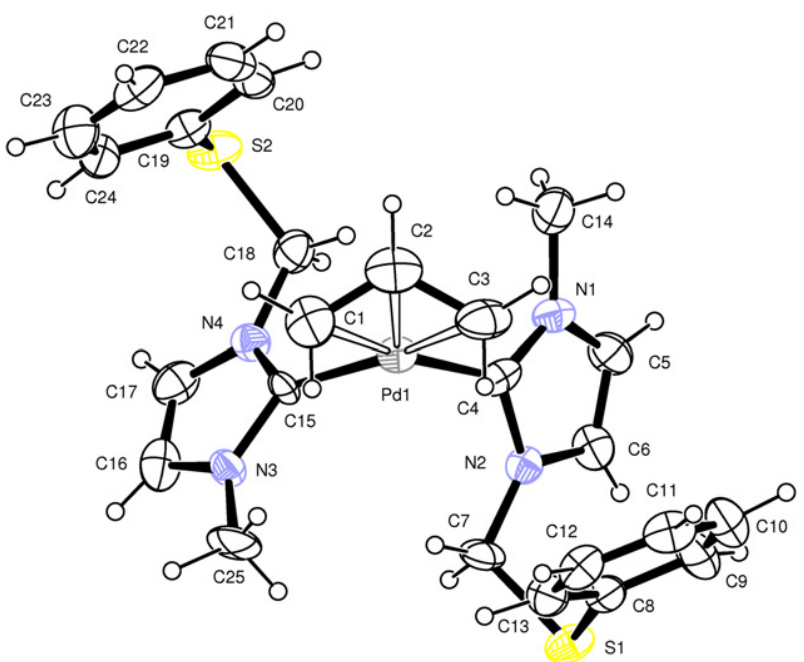

Fig. 3. An ORTEP view of $\mathrm{Pd}(\mathrm{II})$ cationic complex $\mathbf{6 d}$ showing the thermal ellipsoids at $30 \%$ probability level.

in SI together with the dedicated program written in the SCIENTIST $^{\circledR}$ environment.

\subsection{Synthesis of the $N$-arylimidazoles (1)}

The N-arylimidazoles 1b [51], 1d [5n] and 1e [14] are literature products and were synthesized accordingly.

\subsubsection{1-Methyl-3-methylsulfanylmethyl-2,3-dihydro-1H- imidazolium bromide (1a)}

To a suspension of $1.96 \mathrm{~g}$ of $\mathrm{KBr}$ in $100 \mathrm{~mL}$ of anhydrous acetonitrile $0.92 \mathrm{~mL}(11 \mathrm{mmol})$ of chloromethylsulfide and $0.97 \mathrm{~mL}$ (12.1 mmol) of 1-methyl-1H-imidazole were added under inert atmosphere (Ar). The mixture was vigorously stirred for $24 \mathrm{~h}$ and the solvent removed by a rotary evaporator. The residual was dissolved in $\mathrm{CH}_{2} \mathrm{Cl}_{2}(20 \mathrm{~mL})$ and $\mathrm{KBr}$ filtered off on a celite filter. The solution was completely dried under vacuum and the oily residual was washed with diethyl ether and dried under vacuum. $2.1 \mathrm{~g}$ (yield $88 \%$ ) of the title product were obtained as a pale yellow oil.

${ }^{1} \mathrm{H} \mathrm{NMR}\left(\mathrm{CDCl}_{3}, \mathrm{~T}=298 \mathrm{~K}, \mathrm{ppm}\right) \delta: 2.26\left(\mathrm{~s}, 3 \mathrm{H}, \mathrm{S}-\mathrm{CH}_{3}\right), 4.12(\mathrm{~s}$, $\left.3 \mathrm{H}, \mathrm{N}-\mathrm{CH}_{3}\right), 5.58\left(\mathrm{~s}, 2 \mathrm{H}, \mathrm{CH}_{2} \mathrm{~S}\right), 7.59(\mathrm{t}, J=1.7 \mathrm{~Hz}, 1 \mathrm{H}, \mathrm{CH}=\mathrm{CH} \mathrm{Im})$,
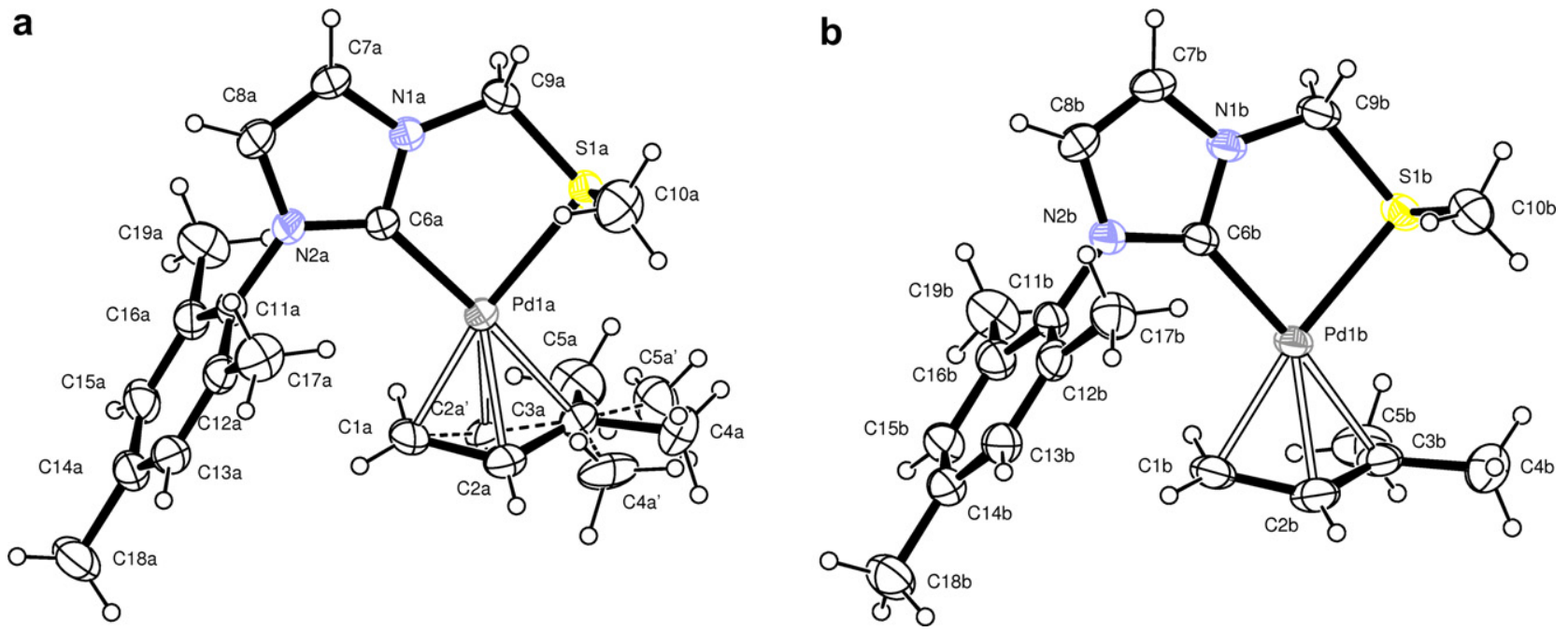

Fig. 2. ORTEP views of both Pd (II) cationic complexes of compound $\mathbf{5 b}$ showing the thermal ellipsoids at 30\% probability level. 
Table 2

Selected bond distances and angles $\left(\AA\right.$ and $\left.{ }^{\circ}\right)$ for $\mathbf{5 b}$.

\begin{tabular}{|c|c|c|}
\hline & A & B \\
\hline \multicolumn{3}{|l|}{ Distances } \\
\hline $\mathrm{Pd} 1-\mathrm{C} 1$ & $2.108(4)$ & $2.131(4)$ \\
\hline $\mathrm{Pd} 1-\mathrm{C} 2$ & $2.132(5)$ & $2.152(3)$ \\
\hline $\mathrm{Pd} 1-\mathrm{C} 2^{\prime}$ & $2.230(11)$ & \\
\hline Pd1-C3 & $2.235(3)$ & $2.235(3)$ \\
\hline $\mathrm{Pd} 1-\mathrm{C} 6$ & $2.026(3)$ & $2.026(3)$ \\
\hline $\mathrm{Pd} 1-\mathrm{S} 1$ & $2.3703(8)$ & $2.3545(9)$ \\
\hline $\mathrm{C} 1-\mathrm{C} 2$ & $1.439(9)$ & $1.459(6)$ \\
\hline $\mathrm{C} 1-\mathrm{C} 2^{\prime}$ & $1.384(14)$ & \\
\hline $\mathrm{C} 2-\mathrm{C} 3$ & $1.363(7)$ & $1.395(5)$ \\
\hline $\mathrm{C} 2^{\prime}-\mathrm{C} 3$ & $1.439(14)$ & \\
\hline C6-N1 & $1.343(4)$ & $1.353(4)$ \\
\hline C6-N2 & $1.348(4)$ & $1.354(4)$ \\
\hline \multicolumn{3}{|l|}{ Angles } \\
\hline $\mathrm{C} 1-\mathrm{Pd} 1-\mathrm{S} 1$ & $169.8(1)$ & $168.3(1)$ \\
\hline $\mathrm{C} 2-\mathrm{Pd} 1-\mathrm{S} 1$ & 131.5(2) & 132.1(1) \\
\hline $\mathrm{C} 2^{\prime}-\mathrm{Pd} 1-\mathrm{S} 1$ & $133.3(49$ & \\
\hline $\mathrm{C} 3-\mathrm{Pd} 1-\mathrm{S} 1$ & $101.5(1)$ & $100.5(1)$ \\
\hline $\mathrm{C} 1-\mathrm{Pd} 1-\mathrm{C} 6$ & $106.5(1)$ & $106.4(1)$ \\
\hline $\mathrm{C} 2-\mathrm{Pd} 1-\mathrm{C} 6$ & $141.2(2)$ & $141.8(1)$ \\
\hline $\mathrm{C} 2^{\prime}-\mathrm{Pd} 1-\mathrm{C} 6$ & $135.8(3)$ & \\
\hline $\mathrm{C} 3-\mathrm{Pd} 1-\mathrm{C} 6$ & $173.2(1)$ & $171.2(1)$ \\
\hline $\mathrm{S} 1-\mathrm{Pd} 1-\mathrm{C} 6$ & 83.6(1) & $83.9(1)$ \\
\hline $\mathrm{C} 1-\mathrm{Pd} 1-\mathrm{C} 2$ & $39.7(2)$ & $39.8(2)$ \\
\hline $\mathrm{C} 1-\mathrm{Pd} 1-\mathrm{C} 2^{\prime}$ & $37.2(4)$ & \\
\hline $\mathrm{C} 1-\mathrm{Pd} 1-\mathrm{C} 3$ & $68.4(1)$ & $68.6(1)$ \\
\hline $\mathrm{C} 2-\mathrm{Pd} 1-\mathrm{C} 3$ & $36.3(2)$ & $37.0(1)$ \\
\hline $\mathrm{C} 2^{\prime}-\mathrm{Pd} 1-\mathrm{C} 3$ & $37.6(3)$ & \\
\hline $\mathrm{C} 1-\mathrm{C} 2-\mathrm{C} 3$ & $121.2(6)$ & 119.1(3) \\
\hline $\mathrm{C} 1-\mathrm{C}^{\prime}-\mathrm{C} 3$ & 119.5(1) & \\
\hline \multicolumn{3}{|l|}{ Dihedral angles } \\
\hline $\mathrm{Pd} 1, \mathrm{C} 1, \mathrm{C} 2, \mathrm{C} 6, \mathrm{~S} 1$, C1,C2,C3,C4,C5 & $86.9(2)$ & $86.2(2)$ \\
\hline $\mathrm{Pd} 1, \mathrm{C} 1, \mathrm{C} 2^{\prime}, \mathrm{C} 6, \mathrm{~S} 1, \mathrm{C} 1, \mathrm{C} 2^{\prime}, \mathrm{C} 3, \mathrm{C} 4^{\prime}, \mathrm{C} 5$ & $85.2(6)$ & \\
\hline
\end{tabular}

$7.74(\mathrm{t}, J=1.7 \mathrm{~Hz}, 1 \mathrm{H}, \mathrm{CH}=\mathrm{CH} \mathrm{Im}), 10.47$ (bs, $1 \mathrm{H}, \mathrm{NCHN}) .{ }^{13} \mathrm{C}\left\{{ }^{1} \mathrm{H}\right\}$ NMR $\left(\mathrm{CDCl}_{3}, T=298 \mathrm{~K}, \mathrm{ppm}\right) \delta: 14.9\left(\mathrm{CH}_{3}, \mathrm{~S}-\mathrm{CH}_{3}\right), 37.0\left(\mathrm{CH}_{3}, \mathrm{~N}-\right.$ $\left.\mathrm{CH}_{3}\right), 52.9\left(\mathrm{CH}_{2}, \mathrm{SCH}_{2}\right), 121.8(\mathrm{CH}, \mathrm{CH}=\mathrm{CH} \mathrm{Im}), 123.7(\mathrm{CH}, \mathrm{CH}=\mathrm{CH}$ Im), 137.4 (C, NCHN).

The following compounds were synthesized by a similar procedure.

Table 3

Selected bond distances and angles $\left(\AA\right.$ and $\left.{ }^{\circ}\right)$ for $\mathbf{6 d}$.

\begin{tabular}{|c|c|}
\hline \multicolumn{2}{|l|}{ Distances } \\
\hline $\mathrm{Pd} 1-\mathrm{C} 1$ & $2.211(11)$ \\
\hline $\mathrm{Pd} 1-\mathrm{C} 2$ & $2.162(6)$ \\
\hline $\mathrm{Pd} 1-\mathrm{C} 3$ & $2.133(12)$ \\
\hline $\mathrm{Pd} 1-\mathrm{C} 4$ & $2.022(11)$ \\
\hline $\mathrm{Pd} 1-\mathrm{C} 15$ & $2.070(9)$ \\
\hline $\mathrm{C} 1-\mathrm{C} 2$ & $1.354(17)$ \\
\hline $\mathrm{C} 2-\mathrm{C} 3$ & $1.434(18)$ \\
\hline $\mathrm{C} 4-\mathrm{N} 1$ & $1.348(14)$ \\
\hline $\mathrm{C} 4-\mathrm{N} 2$ & $1.387(12)$ \\
\hline $\mathrm{C} 15-\mathrm{N} 3$ & $1.366(11)$ \\
\hline C15-N4 & $1.317(14)$ \\
\hline \multicolumn{2}{|l|}{ Angles } \\
\hline $\mathrm{C} 1-\mathrm{Pd} 1-\mathrm{C} 4$ & $168.6(4)$ \\
\hline $\mathrm{C} 2-\mathrm{Pd} 1-\mathrm{C} 4$ & $133.3(4)$ \\
\hline $\mathrm{C} 3-\mathrm{Pd} 1-\mathrm{C} 4$ & $101.1(5)$ \\
\hline $\mathrm{C} 1-\mathrm{Pd} 1-\mathrm{C} 15$ & $96.9(4)$ \\
\hline $\mathrm{C} 2-\mathrm{Pd} 1-\mathrm{C} 15$ & $127.5(4)$ \\
\hline $\mathrm{C} 3-\mathrm{Pd} 1-\mathrm{C} 15$ & $164.3(5)$ \\
\hline $\mathrm{C} 4-\mathrm{Pd} 1-\mathrm{C} 15$ & $94.5(1)$ \\
\hline $\mathrm{C} 1-\mathrm{Pd} 1-\mathrm{C} 2$ & $36.0(4)$ \\
\hline $\mathrm{C} 1-\mathrm{Pd} 1-\mathrm{C} 3$ & $67.6(3)$ \\
\hline $\mathrm{C} 2-\mathrm{Pd} 1-\mathrm{C} 3$ & $39.0(5)$ \\
\hline \multicolumn{2}{|l|}{ Dihedral angle } \\
\hline $\mathrm{Pd} 1, \mathrm{C} 1, \mathrm{C} 3, \mathrm{C} 4, \mathrm{C} 15$ - C1,C2,C3 & $68.2(7)$ \\
\hline
\end{tabular}

4.3.2. 1-(2,6-Diisopropyl-phenyl)-3-methylsulfanylmethyl-2,3dihydro-1H-imidazolium bromide (1c)

White solid. Yield 93\%.

${ }^{1} \mathrm{H} \mathrm{NMR}\left(\mathrm{CDCl}_{3}, T=298 \mathrm{~K}, \mathrm{ppm}\right) \delta: 1.19\left(\mathrm{~d}, J=6.8 \mathrm{~Hz}, 6 \mathrm{H},{ }^{\mathrm{i}} \mathrm{Pr}-\right.$ $\left.\mathrm{CH}_{3}\right), 1.28\left(\mathrm{~d}, J=6.8 \mathrm{~Hz}, 6 \mathrm{H},{ }^{\mathrm{i}} \mathrm{Pr}-\mathrm{CH}_{3}\right), 2.32$ (sept, $2 \mathrm{H}, J=6.8 \mathrm{~Hz},{ }^{\mathrm{i}} \mathrm{Pr}-$ $\mathrm{CH}$ ), 2.37 (s, 3H, S-CH $\mathrm{CH}_{3}, 6.09$ (s, 2H, $\mathrm{CH}_{2} \mathrm{~S}$ ), 7.23 (bt, $1 \mathrm{H}, \mathrm{CH}=\mathrm{CH} \mathrm{Im}$ ), 7.34 (d, $J=7.8 \mathrm{~Hz}, 2 \mathrm{H}, m$-aryl-H), 7.57 (t, $J=7.8 \mathrm{~Hz}, 2 \mathrm{H}, p$-aryl-H), 8.01(bt, $1 \mathrm{H}, \mathrm{CH}=\mathrm{CH}$ Im), 10.82 (bt, $1 \mathrm{H}, \mathrm{NCHN})$.

${ }^{13} \mathrm{C}\left\{{ }^{1} \mathrm{H}\right\} \mathrm{NMR}\left(\mathrm{CDCl}_{3}, \mathrm{~T}=298 \mathrm{~K}, \mathrm{ppm}\right) \delta: 14.3\left(\mathrm{CH}_{3}, \mathrm{~S}-\mathrm{CH}_{3}\right), 23.9$ $\left(\mathrm{CH}_{3},{ }^{\mathrm{i}} \mathrm{Pr}-\mathrm{CH}_{3}\right), 24.4\left(\mathrm{CH}_{3},{ }^{\mathrm{i}} \mathrm{Pr}-\mathrm{CH}_{3}\right), 28.7\left(\mathrm{CH},{ }^{\mathrm{i}} \mathrm{Pr}-\mathrm{CH}\right), 52.7\left(\mathrm{CH}_{2}\right.$, $\left.\mathrm{SCH}_{2}\right), 123.0(\mathrm{CH}, \mathrm{CH}=\mathrm{CH} \mathrm{Im}), 124.4(\mathrm{CH}, \mathrm{CH}=\mathrm{CH} \mathrm{Im}), 124.6(\mathrm{CH}, \mathrm{m}-$ aryl-CH); 129.9 (C, i-aryl-C); $131.9(\mathrm{CH}, p$-aryl-CH); $138.2(\mathrm{CH}$, NCHN); 145.5 (C, o-aryl-C).

\subsubsection{1-Methyl-3-(benzyl)-2,3-dihydro-1H-imidazolium bromide (1f)}

Sticky yellowish oil (washed with diethyl ether and pentane). Yield 92\%.

${ }^{1} \mathrm{H} \mathrm{NMR}\left(\mathrm{CDCl}_{3}, T=298 \mathrm{~K}, \mathrm{ppm}\right) \delta: 3.99\left(\mathrm{~s}, 3 \mathrm{H}, \mathrm{N}-\mathrm{CH}_{3}\right), 5.53(\mathrm{~s}, 2 \mathrm{H}$, $\left.\mathrm{CH}_{2} \mathrm{~N}\right), 7.27-7.32(\mathrm{~m}, 3 \mathrm{H}$, aryl-H), 7.42-7.46 (m, 3H, $\mathrm{CH}=\mathrm{CH}$ Im, aryl$\mathrm{H}), 7.56(\mathrm{t}, J=1.8 \mathrm{~Hz}, 1 \mathrm{H}, \mathrm{CH}=\mathrm{CH} \mathrm{Im}), 10.25(\mathrm{bt}, 1 \mathrm{H}, \mathrm{NCHN}) .{ }^{13} \mathrm{C}\left\{{ }^{1} \mathrm{H}\right\}$ $\mathrm{NMR}\left(\mathrm{CDCl}_{3}, T=298 \mathrm{~K}, \mathrm{ppm}\right) \delta: 36.7\left(\mathrm{CH}_{3}, \mathrm{~N}-\mathrm{CH}_{3}\right), 53.0\left(\mathrm{CH}_{2}, \mathrm{Ph}-\mathrm{CH}_{2}\right)$, $122.0(\mathrm{CH}, \mathrm{CH}=\mathrm{CH} \mathrm{Im}), 123.7(\mathrm{CH}, \mathrm{CH}=\mathrm{CH} \mathrm{Im}), 128.9(\mathrm{CH}, m-\mathrm{Ph})$; $129.2(\mathrm{CH}, o-\mathrm{Ph}) ; 129.3(\mathrm{CH}, p-\mathrm{Ph}) ; 133.0(\mathrm{C}, i-\mathrm{Ph}) ; 136.9(\mathrm{CH}, \mathrm{NCHN})$.

\subsubsection{1-(4-Methyl-benzyl)-3-methyl-2,3-dihydro-1H-imidazolium} bromide (1g)

Sticky yellowish oil (washed with diethyl ether and pentane). Yield 96\%.

${ }^{1} \mathrm{H} \mathrm{NMR}\left(\mathrm{CDCl}_{3}, T=298 \mathrm{~K}, \mathrm{ppm}\right) \delta: 1.34\left(\mathrm{~s}, 3 \mathrm{H}\right.$, tolyl- $\left.\mathrm{CH}_{3}\right), 4.08(\mathrm{~s}$, $\left.3 \mathrm{H}, \mathrm{N}-\mathrm{CH}_{3}\right), 5.52\left(\mathrm{~s}, 2 \mathrm{H}, \mathrm{CH}_{2} \mathrm{~N}\right), 7.19(\mathrm{~d}, J=7.8 \mathrm{~Hz}, 2 \mathrm{H}$, aryl-H), $7.29(\mathrm{t}$, $J=1.8 \mathrm{~Hz}, 1 \mathrm{H}, \mathrm{CH}=\mathrm{CH} \mathrm{Im}), 7.36(\mathrm{~d}, J=7.8 \mathrm{~Hz}, 2 \mathrm{H}, \operatorname{aryl}-\mathrm{H}), 7.43(\mathrm{t}$, $J=1.8 \mathrm{~Hz}, 1 \mathrm{H}, \mathrm{CH}=\mathrm{CH} \mathrm{Im}), 10.55$ (bt, $1 \mathrm{H}, \mathrm{NCHN}) .{ }^{13} \mathrm{C}\left\{{ }^{1} \mathrm{H}\right\} \mathrm{NMR}$ $\left(\mathrm{CDCl}_{3}, \mathrm{~T}=298 \mathrm{~K}, \mathrm{ppm}\right) \delta: 21.1\left(\mathrm{CH}_{3}, \mathrm{Ph}-\mathrm{CH}_{3}\right), 36.7\left(\mathrm{CH}_{3}, \mathrm{~N}-\mathrm{CH}_{3}\right)$, $53.0\left(\mathrm{CH}_{2}, \mathrm{Ph}-\mathrm{CH}_{2}\right), 121.8(\mathrm{CH}, \mathrm{CH}=\mathrm{CH} \mathrm{Im}), 123.6(\mathrm{CH}, \mathrm{CH}=\mathrm{CH} \mathrm{Im})$, $128.9(\mathrm{CH}, m-\mathrm{Ph}) ; 129.9(\mathrm{CH}, o-\mathrm{Ph}) ; 129.9(\mathrm{C}, i-\mathrm{Ph}) ; 137.0(\mathrm{CH}$, NCHN); 139.4 (C, $p-\mathrm{Ph})$.

\subsection{Synthesis of $\mathrm{AgBr}\left(\mathrm{NR}, \mathrm{NCH}_{2} \mathrm{SR}^{\prime}-\mathrm{NHC}\right)$ carbene derivatives}

The N-arylimidazoles $\mathbf{2 b}$ [51], 2d [5n] and 2e [14] are literature products and were synthesized accordingly.

\subsection{1. $\mathrm{AgBr}\left(\mathrm{NMe}, \mathrm{NCH}_{2} \mathrm{SMe}-\mathrm{NHC}\right)(\mathbf{2 a})$}

To $0.4633 \mathrm{~g}$ (2.09 $\mathrm{mmol})$ of $\mathbf{1 a}$ dissolved in $20 \mathrm{~mL}$ of anhydrous $\mathrm{CH}_{2} \mathrm{Cl}_{2}$ under inert atmosphere $(\mathrm{Ar}), 0.2658 \mathrm{~g}$ ( $1.14 \mathrm{mmol}$ ) of $\mathrm{Ag}_{2} \mathrm{O}$ was added, and the resulting mixture was vigorously stirred in the dark for $24 \mathrm{~h}$. The excess of $\mathrm{Ag}_{2} \mathrm{O}$ was eventually removed by filtration on a millipore filter and the resulting clear solution concentrated under vacuum to small volume. Addition of diethyl ether yields the precipitation of a sticky solid which was separated by decantation, washed with some portions of diethyl ether and dried under high vacuum. $0.5125 \mathrm{~g}$ of the title complex was obtained as white solid (yield 75\%).

${ }^{1} \mathrm{H}$ NMR $\left(\mathrm{CDCl}_{3}, T=298 \mathrm{~K}, \mathrm{ppm}\right) \delta: 2.13\left(\mathrm{~s}, 3 \mathrm{H}, \mathrm{S}-\mathrm{CH}_{3}\right), 3.88(\mathrm{~s}$, $\left.3 \mathrm{H}, \mathrm{N}-\mathrm{CH}_{3}\right), 5.16\left(\mathrm{~s}, 2 \mathrm{H}, \mathrm{CH}_{2} \mathrm{~S}\right), 7.05(\mathrm{~d}, J=1.8 \mathrm{~Hz}, 1 \mathrm{H}, \mathrm{CH}=\mathrm{CH} \mathrm{Im})$, $7.25(\mathrm{~d}, J=1.8 \mathrm{~Hz}, 1 \mathrm{H}, \mathrm{CH}=\mathrm{CH} \mathrm{Im}) .{ }^{13} \mathrm{C}\left\{{ }^{1} \mathrm{H}\right\} \mathrm{NMR}\left(\mathrm{CDCl}_{3}, T=298 \mathrm{~K}\right.$, ppm) $\delta: 14.4\left(\mathrm{CH}_{3}, \mathrm{~S}-\mathrm{CH}_{3}\right), 38.9\left(\mathrm{CH}_{3}, \mathrm{~N}-\mathrm{CH}_{3}\right), 54.7\left(\mathrm{CH}_{2}, \mathrm{SCH}_{2}\right)$, $120.4(\mathrm{CH}, \mathrm{CH}=\mathrm{CH} \mathrm{Im}), 123.0(\mathrm{CH}, \mathrm{CH}=\mathrm{CH} \mathrm{Im}), 181.8(\mathrm{C}, \mathrm{NCN})$.

The following complexes were synthesized by a similar procedure.

\subsection{2. $\mathrm{AgBr}\left(\mathrm{Ndi}\right.$-i-propylphenyl, $\left.\mathrm{NCH}_{2} \mathrm{SMe}-\mathrm{NHC}\right)(\mathbf{2 c})$ \\ White solid. Yield 64\%.}

${ }^{1} \mathrm{H} \mathrm{NMR}\left(\mathrm{CDCl}_{3}, T=298 \mathrm{~K}, \mathrm{ppm}\right) \delta: 1.15\left(\mathrm{~d}, J=6.8 \mathrm{~Hz}, 6 \mathrm{H},{ }^{\mathrm{i}} \mathrm{Pr}-\right.$ $\left.\mathrm{CH}_{3}\right), 1.25\left(\mathrm{~d}, \mathrm{~J}=6.8 \mathrm{~Hz}, 6 \mathrm{H},{ }^{\mathrm{i}} \mathrm{Pr}-\mathrm{CH}_{3}\right), 2.17$ (s, 3H, S-CH$)_{3}, 2.38$ (sept, 
$\left.2 \mathrm{H}, J=6.8 \mathrm{~Hz},{ }^{\mathrm{i}} \mathrm{Pr}-\mathrm{CH}\right), 5.29\left(\mathrm{~s}, 2 \mathrm{H}, \mathrm{CH}_{2} \mathrm{~S}\right), 7.09(\mathrm{~d}, J=1.7 \mathrm{~Hz}, 1 \mathrm{H}$, $\mathrm{CH}=\mathrm{CH} \mathrm{Im}), 7.09(\mathrm{~d}, J=7.7 \mathrm{~Hz}, 2 \mathrm{H}, \mathrm{m}$-aryl-H), $7.47(\mathrm{~d}, J=1.7 \mathrm{~Hz}, 1 \mathrm{H}$, $\mathrm{CH}=\mathrm{CH} \mathrm{Im}), 7.50\left(\mathrm{t}, J=7.7 \mathrm{~Hz}, 2 \mathrm{H}, p\right.$-aryl-H). ${ }^{13} \mathrm{C}\left\{{ }^{1} \mathrm{H}\right\} \mathrm{NMR}\left(\mathrm{CDCl}_{3}\right.$, $T=298 \mathrm{~K}, \mathrm{ppm}) \delta: 14.1\left(\mathrm{CH}_{3}, \mathrm{~S}-\mathrm{CH}_{3}\right), 24.1\left(\mathrm{CH}_{3},{ }^{\mathrm{i}} \mathrm{Pr}-\mathrm{CH}_{3}\right), 24.5\left(\mathrm{CH}_{3}\right.$, $\left.{ }^{\mathrm{i}} \mathrm{Pr}-\mathrm{CH}_{3}\right), 28.3\left(\mathrm{CH},{ }^{\mathrm{i}} \mathrm{Pr}-\mathrm{CH}\right), 54.7\left(\mathrm{CH}_{2}, \mathrm{SCH}_{2}\right), 120.0(\mathrm{CH}, \mathrm{CH}=\mathrm{CH} \mathrm{Im})$, $124.3(\mathrm{CH}, m$-aryl-CH$) ; 125.0(\mathrm{CH}, \mathrm{CH}=\mathrm{CH} \mathrm{Im}), 130.6(\mathrm{CH}, p$-aryl$\mathrm{CH}) ; 134.3$ (C, $i$-aryl-C); 145.5 (C, o-aryl-C); $183.5\left(\mathrm{~d}, J_{\mathrm{AgC}}=271 \mathrm{~Hz}\right.$, C, NCN).

\subsection{3. $\mathrm{AgBr}\left(\mathrm{NMe}, \mathrm{NCH}_{2} \mathrm{Ph}-\mathrm{NHC}\right)(\mathbf{2 f})$}

White solid. Yield $91 \%$.

${ }^{1} \mathrm{H} \mathrm{NMR}\left(\mathrm{CDCl}_{3}, T=298 \mathrm{~K}, \mathrm{ppm}\right) \delta: 3.86\left(\mathrm{~s}, 3 \mathrm{H}, \mathrm{N}-\mathrm{CH}_{3}\right), 5.30(\mathrm{~s}$, $\left.2 \mathrm{H}, \mathrm{CH}_{2} \mathrm{~N}\right), 6.94(\mathrm{~d}, J=1.8 \mathrm{~Hz}, 1 \mathrm{H}, \mathrm{CH}=\mathrm{CH} \mathrm{Im}), 6.99(\mathrm{~d}, J=1.8 \mathrm{~Hz}$, $1 \mathrm{H}, \mathrm{CH}=\mathrm{CH}$ Im), 7.24-7.28 (m, 2H, aryl-H), 7.32-7.39 (m, 3H, arylH). ${ }^{13} \mathrm{C}\left\{{ }^{1} \mathrm{H}\right\} \mathrm{NMR}\left(\mathrm{CDCl}_{3}, \mathrm{~T}=298 \mathrm{~K}, \mathrm{ppm}\right) \delta: 38.8\left(\mathrm{CH}_{3}, \mathrm{~N}-\mathrm{CH}_{3}\right), 55.6$ $\left(\mathrm{CH}_{2}, \mathrm{Ph}-\mathrm{CH}_{2}\right), 121.0(\mathrm{CH}, \mathrm{CH}=\mathrm{CH} \mathrm{Im}), 122.5(\mathrm{CH}, \mathrm{CH}=\mathrm{CH}$ Im $), 127.7$ (CH, m-Ph); 129.1 (CH, o-Ph); 129.4 (CH, p-Ph); 135.3 (C, i-Ph); 181.2 (C, NCN).

\subsection{4. $\mathrm{AgBr}\left(\mathrm{NMe}, \mathrm{NCH}_{2} \mathrm{Tol}-\mathrm{NHC}\right)(\mathbf{2 g})$ \\ White solid. Yield $88 \%$.}

${ }^{1} \mathrm{H}$ NMR $\left(\mathrm{CDCl}_{3}, T=298 \mathrm{~K}, \mathrm{ppm}\right) \delta: 2.35\left(\mathrm{~s}, 3 \mathrm{H}\right.$, tolyl- $\left.\mathrm{CH}_{3}\right), 3.86(\mathrm{~s}$, $\left.3 \mathrm{H}, \mathrm{N}-\mathrm{CH}_{3}\right), 5.24\left(\mathrm{~s}, 2 \mathrm{H}, \mathrm{CH}_{2} \mathrm{~N}\right), 6.92(\mathrm{~d}, J=1.8 \mathrm{~Hz}, 1 \mathrm{H}, \mathrm{CH}=\mathrm{CH} \mathrm{Im})$, $6.97(\mathrm{~d}, J=1.8 \mathrm{~Hz}, 1 \mathrm{H}, \mathrm{CH}=\mathrm{CH} \mathrm{Im}), 7.15\left(\mathrm{~m}, 4 \mathrm{H}\right.$, aryl-H). ${ }^{13} \mathrm{C}\left\{{ }^{1} \mathrm{H}\right\}$ NMR $\left(\mathrm{CDCl}_{3}, T=298 \mathrm{~K}, \mathrm{ppm}\right) \delta: 21.1\left(\mathrm{CH}_{3}, \mathrm{Ph}-\mathrm{CH}_{3}\right), 38.7\left(\mathrm{CH}_{3}, \mathrm{~N}-\right.$ $\left.\mathrm{CH}_{3}\right), 55.4\left(\mathrm{CH}_{2}, \mathrm{Ph}-\mathrm{CH}_{2}\right), 120.9(\mathrm{CH}, \mathrm{CH}=\mathrm{CH} \mathrm{Im}), 122.4(\mathrm{CH}, \mathrm{CH}=$ CH Im), 127.8 (CH, $m-\mathrm{Ph}) ; 129.7$ (CH, o-Ph); 132.3 (C, $i-\mathrm{Ph}) ; 145.5$ (C, p-Ph); 181.5 (C, NCN).

\subsection{Synthesis of the palladium allyl carbene complexes (3)}

\subsection{1. $\left[\mathrm{Pd}\left(\eta^{3}\right.\right.$-allyl $\left.)\left(\mathrm{NMe}, \mathrm{NCH}_{2} \mathrm{SMe}-\mathrm{NHC}\right) \mathrm{Cl}\right](\mathbf{3 a})$}

To $0.0389 \mathrm{~g}(0.105 \mathrm{mmol})$ of $\left[\mathrm{Pd}\left(\eta^{3}\right.\right.$-allyl $) \mathrm{Cl}_{2}$ [27] dissolved in $20 \mathrm{~mL}$ of anhydrous $\mathrm{CH}_{2} \mathrm{Cl}_{2}, 0.0692 \mathrm{~g}(0.21 \mathrm{mmol})$ of complex 2a was added under inert atmosphere $(\mathrm{Ar})$. The reaction mixture was stirred for $1 \mathrm{~h}$ and the $\mathrm{AgBr}$ filtered off on a millipore filter. The clear solution was concentrated under vacuum. Addition of diethyl ether yields the precipitation of $0.0273 \mathrm{~g}$ (yield $40 \%$ ) of the title compounds as a whitish sticky solid which was washed several times with diethyl ether.

${ }^{1} \mathrm{H} \mathrm{NMR}\left(\mathrm{CDCl}_{3}, T=298 \mathrm{~K}, \mathrm{ppm}\right) \delta: 2.15$ (s, 3H, S-CH$), 2.52$ (bd, $1 \mathrm{H}$, anti allyl- $\mathrm{H}$ trans-Cl), $3.34(\mathrm{~d}, J=14.0 \mathrm{~Hz}, 1 \mathrm{H}$, anti allyl-H transC), 3.51 (bs, $1 \mathrm{H}$, syn allyl-H trans-Cl), $3.84\left(\mathrm{~s}, 3 \mathrm{H}, \mathrm{N}-\mathrm{CH}_{3}\right), 4.36$ (d, $J=7.4 \mathrm{~Hz}, 1 \mathrm{H}$, syn allyl-H trans-C), 5.29 (bs $2 \mathrm{H}, \mathrm{CH}_{2} \mathrm{~S}$ ), 5.37 (bm, $1 \mathrm{H}$, central-allyl-H), 6.98 (bd, $1 \mathrm{H}, \mathrm{CH}=\mathrm{CH}$ Im). 7.24 (bd, $1 \mathrm{H}, \mathrm{CH}=\mathrm{CH} \mathrm{Im}$ ).

The following complexes were synthesized similarly.

\subsection{2. $\left[\mathrm{Pd}\left(\eta^{3}\right.\right.$-allyl)(NMe, $\left.\left.\mathrm{NCH}_{2} \mathrm{SPh}-\mathrm{NHC}\right) \mathrm{Cl}\right](\mathbf{3} \boldsymbol{f})$}

Whitish solid. Yield $88 \%$.

${ }^{1} \mathrm{H} \mathrm{NMR}\left(\mathrm{CDCl}_{3}, T=298 \mathrm{~K}, \mathrm{ppm}\right) \delta: 2.21(\mathrm{~d}, J=12.3 \mathrm{~Hz}, 1 \mathrm{H}$, anti allyl-H trans-Cl), 3.22-3.26 (m, $2 \mathrm{H}$, anti allyl-H trans-C, syn allyl-H trans-Cl), $3.85\left(\mathrm{~s}, 3 \mathrm{H}, \mathrm{N}-\mathrm{CH}_{3}\right), 4.28$ (dd, $J=7.6,2.2 \mathrm{~Hz}, 1 \mathrm{H}$, syn allyl-H trans-C), 5.23 (bm, $1 \mathrm{H}$, central-allyl-H), 5.44 (broad AB system, $2 \mathrm{H}$, $\mathrm{CH}_{2} \mathrm{~S}$ ), 6.89 (d, $\left.J=1.9 \mathrm{~Hz}, 1 \mathrm{H}, \mathrm{CH}=\mathrm{CH} \mathrm{Im}\right), 6.94(\mathrm{~d}, J=1.9 \mathrm{~Hz}, 1 \mathrm{H}$, $\mathrm{CH}=\mathrm{CH} \mathrm{Im}), 7.25-7.37$ ( $\mathrm{m}, 5 \mathrm{H}, \mathrm{SPh})$.

\subsection{3. $\left[\mathrm{Pd}\left(\eta^{3}\right.\right.$-allyl)(NMe, $\left.\left.\mathrm{NCH}_{2} \mathrm{Tol}-\mathrm{NHC}\right) \mathrm{Cl}\right]$ (3g)}

Whitish solid. Yield $77 \%$.

${ }^{1} \mathrm{H} \mathrm{NMR}\left(\mathrm{CDCl}_{3}, T=298 \mathrm{~K}, \mathrm{ppm}\right) \delta: 2.25(\mathrm{~d}, J=12.5 \mathrm{~Hz}, 1 \mathrm{H}$, anti allyl-H trans-Cl), 2.34 (s, 3H, tolyl- $\left.\mathrm{CH}_{3}\right), 3.26-3.28(\mathrm{~m}, 2 \mathrm{H}$, anti allyl$\mathrm{H}$ trans-C and syn allyl-H trans- $\mathrm{Cl}), 3.85\left(\mathrm{~s}, 3 \mathrm{H}, \mathrm{N}-\mathrm{CH}_{3}\right), 4.30(\mathrm{~d}$, $J=7.7 \mathrm{~Hz}, J=2.2 \mathrm{~Hz}, 1 \mathrm{H}$, anti allyl-H trans-C), 5.26 (bm, $1 \mathrm{H}$, central allyl-H), 5.35, 5.43 (AB system, $J=15.0 \mathrm{~Hz}, 2 \mathrm{H}, \mathrm{CH}_{2} \mathrm{~N}$ ), 6.86 (d, $J=1.9 \mathrm{~Hz}, 1 \mathrm{H}, \mathrm{CH}=\mathrm{CH} \mathrm{Im}), 6.92(\mathrm{~d}, J=1.9 \mathrm{~Hz}, 1 \mathrm{H}, \mathrm{CH}=\mathrm{CH} \mathrm{Im}), 7.16$ (m, $4 \mathrm{H}$, tolyl-H).

\subsection{Synthesis of the palladium allyl carbene complexes (4)}

\subsection{1. $\left[\mathrm{Pd}\left(\eta^{3}\right.\right.$-allyl $\left.)\left(\mathrm{NMe}, \mathrm{NCH}_{2} \mathrm{SMe}^{-\mathrm{NHC}}\right)\right] \mathrm{ClO}_{4}(\mathbf{4 a})$}

To $0.0255 \mathrm{~g}(0.069 \mathrm{mmol})$ of $\left[\mathrm{Pd}\left(\eta^{3} \text {-allyl }\right) \mathrm{Cl}\right]_{2}$ dissolved in anhydrous $\mathrm{CH}_{2} \mathrm{Cl}_{2}(20 \mathrm{~mL})$ under inert atmosphere (Ar), 0.0452 $(0.137 \mathrm{mmol})$ of the silver complex 2a was added. Precipitation of $\mathrm{AgBr}$ as a cloudy suspension was immediately noticed and after $1 \mathrm{~h}$ the reaction mixture was filtered off on a millipore filter. The clear solution was treated with $0.0392 \mathrm{~g}(0.279 \mathrm{mmol})$ of monohydrated $\mathrm{NaClO}_{4}$ dissolved in $\mathrm{MeOH}(7 \mathrm{~mL})$. The reaction mixture was stirred for $30 \mathrm{~min}$ and the solvent removed by a rotary evaporator. The residue was treated with anhydrous $\mathrm{CH}_{2} \mathrm{Cl}_{2}$ $(10 \mathrm{~mL})$ and the $\mathrm{NaCl}$ filtered off. The resulting clear solution was reduced to small volume and the title product was precipitated by addition of diethyl ether, filtered off and dried under high vacuum. $0.0394 \mathrm{~g}$ (yield $84 \%$ ) of the title complex as white solid was obtained.

${ }^{1} \mathrm{H}$ NMR $\left(\mathrm{CDCl}_{3}, T=298 \mathrm{~K}, \mathrm{ppm}\right) \delta: 2.64\left(\mathrm{~s}, 3 \mathrm{H}, \mathrm{S}-\mathrm{CH}_{3}\right), 3.29(\mathrm{bd}$, $2 \mathrm{H}$, anti allyl-H trans-S, anti allyl-H trans-C), $3.88\left(\mathrm{~s}, 3 \mathrm{H}, \mathrm{N}-\mathrm{CH}_{3}\right)$, 4.54 (bd, $1 \mathrm{H}$, syn allyl-H trans-S), 4.59 (bd, $1 \mathrm{H}$, syn allyl-H trans-C), 5.32 (broad AB system, $2 \mathrm{H}, \mathrm{CH}_{2} \mathrm{~S}$ ), 5.52 ( $\mathrm{m}, 1 \mathrm{H}$, central-allyl-H), 7.16 $(\mathrm{d}, J=1.8 \mathrm{~Hz}, 1 \mathrm{H}, \mathrm{CH}=\mathrm{CH} \mathrm{Im}) .7 .59(\mathrm{~d}, J=1.8 \mathrm{~Hz}, 1 \mathrm{H}, \mathrm{CH}=\mathrm{CH} \mathrm{Im})$.

${ }^{13} \mathrm{C}\left\{{ }^{1} \mathrm{H}\right\}$ NMR $\left(\mathrm{CDCl}_{3}, T=298 \mathrm{~K}, \mathrm{ppm}\right) \delta: 20.9\left(\mathrm{CH}_{3}, \mathrm{~S}-\mathrm{CH}_{3}\right), 39.5$ $\left(\mathrm{CH}_{3}, \mathrm{~N}-\mathrm{CH}_{3}\right), 55.7\left(\mathrm{CH}_{2}, \mathrm{SCH}_{2}\right), 58.7\left(\mathrm{CH}_{2}\right.$, allyl trans-S $), 69.3\left(\mathrm{CH}_{2}\right.$, allyl trans-C), $118.9(\mathrm{CH}$, central allyl), $120.9(\mathrm{CH}, \mathrm{CH}=\mathrm{CH} \mathrm{Im}), 123.8$ $(\mathrm{CH}, \mathrm{CH}=\mathrm{CH}$ Im), 179.3 (C, NCN).

IR ( $\mathrm{KBr}$ pellet, $\mathrm{cm}^{-1}$ ): 1092 (ClO stretching), 623 (ClO bending).

Anal. Calcd. for $\mathrm{C}_{9} \mathrm{H}_{15} \mathrm{ClN}_{2} \mathrm{O}_{4} \mathrm{PdS}$ : C, 27.78; $\mathrm{H}, 3.89 ; \mathrm{N}, 7.20$. Found: C, 27.91; H, 3.77; N, 7.27\%.

The following complexes were synthesized by a similar procedure.

\subsection{2. $\left[\mathrm{Pd}\left(\eta^{3}\right.\right.$-allyl)(NMesityl, $\left.\mathrm{NCH}_{2} \mathrm{SMe}^{-\mathrm{NHC})}\right] \mathrm{ClO}_{4}$ (4b)}

White solid. Yield $72 \%$.

${ }^{1} \mathrm{H}$ NMR $\left(\mathrm{CDCl}_{3}, T=298 \mathrm{~K}, \mathrm{ppm}\right) \delta: 1.93(\mathrm{~s}, 3 \mathrm{H}, o$-aryl-CH 3$)$, $2.02\left(\mathrm{~s}, 3 \mathrm{H}, o\right.$-aryl- $\left.\mathrm{CH}_{3}\right), 2.40\left(\mathrm{~s}, 3 \mathrm{H}, p\right.$-aryl- $\left.\mathrm{CH}_{3}\right), 2.48(\mathrm{~d}, J=13.6$, $1 \mathrm{H}$, anti allyl-H trans-S), $2.72\left(\mathrm{~s}, 3 \mathrm{H}, \mathrm{S}-\mathrm{CH}_{3}\right), 2.93(\mathrm{~d}, J=6.8 \mathrm{~Hz}, 1 \mathrm{H}$, syn allyl-H trans-S), 3.08 (d, $J=13.8 \mathrm{~Hz}, 1 \mathrm{H}$, anti allyl-H trans-C), $4.43(\mathrm{dd}, J=7.7,2.1 \mathrm{~Hz}, 1 \mathrm{H}$, syn allyl-H trans-C), $5.21(\mathrm{~m}, 1 \mathrm{H}$, central-allyl-H), 5.57 (broad $\mathrm{AB}$ system, $\left.2 \mathrm{H}, \mathrm{CH}_{2} \mathrm{~S}\right), 7.04(\mathrm{~s}, 2 \mathrm{H}, m-$ aryl-H), 7.08 (d, $J=1.9 \mathrm{~Hz}, 1 \mathrm{H}, \mathrm{CH}=\mathrm{CH} \mathrm{Im}), 7.88(\mathrm{~d}, J=1.9 \mathrm{~Hz}, 1 \mathrm{H}$, $\mathrm{CH}=\mathrm{CH}$ Im).

${ }^{13} \mathrm{C}\left\{{ }^{1} \mathrm{H}\right\}$ NMR $\left(\mathrm{CDCl}_{3}, T=298 \mathrm{~K}, \mathrm{ppm}\right) \delta: 17.6\left(\mathrm{CH}_{3}, o\right.$-mesityl$\left.\mathrm{CH}_{3}\right), 21.1\left(\mathrm{CH}_{3}, p\right.$-mesityl- $\left.\mathrm{CH}_{3}\right), 21.3\left(\mathrm{CH}_{3}, \mathrm{~S}-\mathrm{CH}_{3}\right), 56.3\left(\mathrm{CH}_{2}, \mathrm{SCH}_{2}\right)$, $59.2\left(\mathrm{CH}_{2}\right.$, allyl trans-S $), 68.3\left(\mathrm{CH}_{2}\right.$, allyl trans-C), $119.0(\mathrm{CH}$, central allyl), $121.7(\mathrm{CH}, \mathrm{CH}=\mathrm{CH} \mathrm{Im}), 123.3(\mathrm{CH}, \mathrm{CH}=\mathrm{CH} \mathrm{Im}), 129.0(\mathrm{CH}, m-$ mesityl-CH); 129.1 ( $\mathrm{CH}$, m-mesityl-CH); 134.7 (C, o-mesityl-C); 135.0 (C, o-mesityl-C); 136.6 (C, i-mesityl-C); 139.9 (C, p-mesityl-C); 180.5 (C, NCN).

IR ( $\mathrm{KBr}$ pellet, $\mathrm{cm}^{-1}$ ): 1102 (ClO stretching), 623 (ClO bending). Anal. Calcd. for $\mathrm{C}_{17} \mathrm{H}_{23} \mathrm{ClN}_{2} \mathrm{O}_{4} \mathrm{PdS}$ : C, 41.39; $\mathrm{H}, 4.70 ; \mathrm{N}, 5.68$. Found: C, 41.51; H, 4.61; N, 5.76\%.

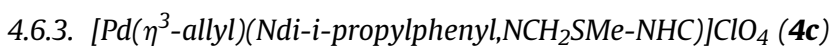

White solid. Yield $90 \%$.

${ }^{1} \mathrm{H}$ NMR $\left(\mathrm{CDCl}_{3}, T=298 \mathrm{~K}, \mathrm{ppm}\right) \delta: 1.07\left(\mathrm{~d}, J=6.8 \mathrm{~Hz}, 3 \mathrm{H},{ }^{\mathrm{i}} \mathrm{Pr}-\right.$ $\left.\mathrm{CH}_{3}\right), 1.15\left(\mathrm{~d}, J=6.8 \mathrm{~Hz}, 3 \mathrm{H},{ }^{\mathrm{i}} \mathrm{Pr}-\mathrm{CH}_{3}\right), 1.18\left(\mathrm{~d}, J=6.8 \mathrm{~Hz}, 6 \mathrm{H},{ }^{\mathrm{i}} \mathrm{Pr}^{-\mathrm{CH}_{3}}\right)$, 2.27 (sept, $1 \mathrm{H}, J=6.8 \mathrm{~Hz}$, $\mathrm{i} P r-\mathrm{CH}), 2.44$ (d, $J=13.9,1 \mathrm{H}$, anti allyl-H trans-S), 2.48 (sept, $1 \mathrm{H}, J=6.8 \mathrm{~Hz},{ }^{\mathrm{i}} \mathrm{Pr}-\mathrm{CH}$ ), 2.69 (s, 3H, S-CH $\mathrm{CH}_{3}, 2.75$ (bd, $J=6.4 \mathrm{~Hz}, 1 \mathrm{H}$, syn allyl-H trans-S), $3.04(\mathrm{~d}, J=13.7 \mathrm{~Hz}, 1 \mathrm{H}$, anti allyl-H trans-C), 4.41 (dd, $J=7.5,2.3 \mathrm{~Hz}, 1 \mathrm{H}$, syn allyl-H trans-C), 5.12 ( $\mathrm{m}, 1 \mathrm{H}$, central-allyl-H), $5.63\left(\mathrm{bs}, 2 \mathrm{H}, \mathrm{CH}_{2} \mathrm{~S}\right), 7.16(\mathrm{~d}, J=1.9 \mathrm{~Hz}, 1 \mathrm{H}$, $\mathrm{CH}=\mathrm{CH}$ Im), $7.32(\mathrm{~d}, J=8.1 \mathrm{~Hz}, 1 \mathrm{H}, m$-aryl-H), $7.33(\mathrm{~d}, J=8.1 \mathrm{~Hz}, 1 \mathrm{H}$, $m$-aryl-H), 7.56 (t, $J=8.1 \mathrm{~Hz}, 1 \mathrm{H}, p$-aryl-H), $7.94(\mathrm{~d}, J=1.9 \mathrm{~Hz}, 1 \mathrm{H}$, $\mathrm{CH}=\mathrm{CH}$ Im). 
${ }^{13} \mathrm{C}\left\{{ }^{1} \mathrm{H}\right\}$ NMR $\left(\mathrm{CDCl}_{3}, T=298 \mathrm{~K}, \mathrm{ppm}\right) \delta: 21.2\left(\mathrm{CH}_{3}, \mathrm{~S}-\mathrm{CH}_{3}\right), 23.4$ $\left(\mathrm{CH}_{3},{ }^{\mathrm{i}} \mathrm{Pr}-\mathrm{CH}_{3}\right), 23.7\left(\mathrm{CH}_{3},{ }^{\mathrm{i}} \mathrm{Pr}-\mathrm{CH}_{3}\right), 24.3\left(\mathrm{CH}_{3},{ }^{\mathrm{i}} \mathrm{Pr}-\mathrm{CH}_{3}\right), 24.7\left(\mathrm{CH}_{3}\right.$, $\left.{ }^{\mathrm{i}} \mathrm{Pr}-\mathrm{CH}_{3}\right), 28.2\left(\mathrm{CH},{ }^{\mathrm{i}} \mathrm{Pr}-\mathrm{CH}\right), 28.3\left(\mathrm{CH}_{3},{ }^{\mathrm{i}} \mathrm{Pr}-\mathrm{CH}_{3}\right), 56.3\left(\mathrm{CH}_{2}, \mathrm{SCH}_{2}\right)$, $60.5\left(\mathrm{CH}_{2}\right.$, allyl trans-S), $68.0\left(\mathrm{CH}_{2}\right.$, allyl trans- $\left.\mathrm{C}\right), 118.8(\mathrm{CH}$, central allyl), $121.6(\mathrm{CH}, \mathrm{CH}=\mathrm{CH}$ Im), $123.9(\mathrm{CH}, m$-aryl-CH); $124.0(\mathrm{CH}, m-$ aryl-CH); $124.5(\mathrm{CH}, \mathrm{CH}=\mathrm{CH} \mathrm{Im}), 130.7(\mathrm{CH}, p$-aryl-CH$) ; 136.4(\mathrm{C}, i-$ aryl-C); 145.6 (C, o-aryl-C); 145.7 (C, o-aryl-C); 181.3 (C, NCN).

IR ( $\mathrm{KBr}$ pellet, $\mathrm{cm}^{-1}$ ): 1089 (ClO stretching), 623 (ClO bending). Anal. Calcd. for $\mathrm{C}_{20} \mathrm{H}_{29} \mathrm{ClN}_{2} \mathrm{O}_{4} \mathrm{PdS}$ : C, 44.87; $\mathrm{H}, 5.46$; N, 5.23. Found: C, 44.94; H, 5.37; N, 5.14\%.

\subsection{4. $\left[\mathrm{Pd}\left(\eta^{3}\right.\right.$-allyl $\left.)\left(\mathrm{NMe}, \mathrm{NCH}_{2} \mathrm{SPh}-\mathrm{NHC}\right)\right] \mathrm{ClO}_{4}(\mathbf{4 d})$}

White solid. Yield $87 \%$.

${ }^{1} \mathrm{H}$ NMR $\left(\mathrm{CD}_{2} \mathrm{Cl}_{2}, T=298 \mathrm{~K}, \mathrm{ppm}\right) \delta: 3.33(\mathrm{~d}, J=13.6 \mathrm{~Hz}, 1 \mathrm{H}$, anti allyl-H trans-C), 3.43 (d, $J=12.5 \mathrm{~Hz}, 1 \mathrm{H}$, anti allyl-H trans-S), 3.93 (s, $\left.3 \mathrm{H}, \mathrm{NCH}_{3}\right), 4.62(\mathrm{dd}, J=7.6, J=2.2,1 \mathrm{H}$, syn allyl-H trans-S), $4.73(\mathrm{dt}$, $J=7.0,1.9 \mathrm{~Hz}, 1 \mathrm{H}$, syn allyl-H trans-C), 5.33, 5.43 (AB system, $\left.J=12.9 \mathrm{~Hz}, 2 \mathrm{H}, \mathrm{CH}_{2} \mathrm{~S}\right), 5.62(\mathrm{~m}, 1 \mathrm{H}$, central-allyl-H), 7.23 (d, $J=1.9 \mathrm{~Hz}, 1 \mathrm{H}, \mathrm{CH}=\mathrm{CH} \mathrm{Im}), 7.46(\mathrm{~d}, J=1.9 \mathrm{~Hz}, 1 \mathrm{H}, \mathrm{CH}=\mathrm{CH} \mathrm{Im})$, 7.43-751 (m, 3H, SPh-H), 7.57-7.61 (m, 2H, SPh-H).

${ }^{13} \mathrm{C}\left\{{ }^{1} \mathrm{H}\right\}$ NMR $\left(\mathrm{CD}_{2} \mathrm{Cl}_{2}, T=298 \mathrm{~K}, \mathrm{ppm}\right) \delta: 39.6\left(\mathrm{CH}_{3}, \mathrm{CH}_{3}-\mathrm{Im}\right)$, 59.4 $\left(\mathrm{CH}_{2}, \mathrm{CH}_{2}-\mathrm{SPh}\right), 59.8\left(\mathrm{CH}_{2}\right.$ allyl trans- $\left.\mathrm{S}\right), 70.6\left(\mathrm{CH}_{2}\right.$ allyl trans- $\left.\mathrm{C}\right)$, 119.4 (CH allyl), $120.0(\mathrm{CH}, \mathrm{CH}=\mathrm{CH} \mathrm{Im}), 124.3(\mathrm{CH}, \mathrm{CH}=\mathrm{CH} \mathrm{Im})$, 129.7 (C, i-Ph), 130.1 (C, o-Ph), 131.0 (C, p-Ph), 132.1 (C, $m-\mathrm{Ph}), 179.2$ (C, Im).

IR ( $\mathrm{KBr}$ pellet, $\mathrm{cm}^{-1}$ ): 1082 ( $\mathrm{ClO}$ stretching), 623 (ClO bending). Anal. Calcd. for $\mathrm{C}_{14} \mathrm{H}_{17} \mathrm{ClN}_{2} \mathrm{O}_{4} \mathrm{PdS}$ : C, 37.26; $\mathrm{H}, 3.80 ; \mathrm{N}, 6.21$. Found: C, 37.37; H, 3.69; N, 6.12\%.

4.6.5. $\left[\mathrm{Pd}\left(\eta^{3}\right.\right.$-allyl) $\left.\left(\mathrm{NMe}, \mathrm{NCH}_{2} \mathrm{Py}-\mathrm{NHC}\right)\right] \mathrm{ClO}_{4}(\mathbf{4 e})$ was synthesized according to a published procedure [8c]

The synthesis of the following complexes is similar to that of the unsubstituted allyl derivative and was carried out starting from $\left[\operatorname{Pd}\left(\eta^{3}-1,1-\mathrm{Me}_{2} \text { allyl }\right)(\mu-\mathrm{Cl})\right]_{2}[28]$.

\subsection{Synthesis of the palladium Me-allyl carbene complexes (5)}

\subsection{1. $\left[\mathrm{Pd}\left(\eta^{3}-1,1-\mathrm{Me}_{2}\right.\right.$ allyl $\left.)\left(\mathrm{NMe}, \mathrm{NCH}_{2} \mathrm{SMe}^{-\mathrm{NHC}}\right)\right] \mathrm{ClO}_{4}$ (5a)}

Whitish solid. Yield 69\%.

${ }^{1} \mathrm{H}$ NMR $\left(\mathrm{CDCl}_{3}, T=298 \mathrm{~K}, \mathrm{ppm}\right) \delta: 1.47\left(\mathrm{~s}, 3 \mathrm{H}, \mathrm{CH}_{3}\right.$ anti allyl$\left.\mathrm{CH}_{3}\right) ; 2.04$ (s, 3H, $\mathrm{CH}_{3}$ syn allyl- $\left.\mathrm{CH}_{3}\right) ; 2.53$ (s, 3H, S-CH $), 3.16$ (dd, $\mathrm{J}=12.7,2.1 \mathrm{~Hz}, 1 \mathrm{H}$, anti allyl-H), $3.87\left(\mathrm{~s}, 3 \mathrm{H}, \mathrm{N}-\mathrm{CH}_{3}\right), 4.59$ (dd, $J=7.6$, $2.1 \mathrm{~Hz}, 1 \mathrm{H}$, syn allyl-H), 5.14 (dd, $J=12.7,7.6 \mathrm{~Hz}, 1 \mathrm{H}$, central-allyl-H), 5.28 (broad AB system, $\left.2 \mathrm{H}, \mathrm{CH}_{2} \mathrm{~S}\right), 7.17(\mathrm{~d}, J=1.8 \mathrm{~Hz}, 1 \mathrm{H}, \mathrm{CH}=\mathrm{CH}$ $\mathrm{Im}), 7.56(\mathrm{~d}, J=1.8 \mathrm{~Hz}, 1 \mathrm{H}, \mathrm{CH}=\mathrm{CH} \mathrm{Im}) .{ }^{13} \mathrm{C}\left\{{ }^{1} \mathrm{H}\right\} \mathrm{NMR}\left(\mathrm{CDCl}_{3}\right.$, $T=298 \mathrm{~K}, \mathrm{ppm}) \delta: 20.0\left(\mathrm{CH}_{3}\right.$, syn allyl- $\left.\mathrm{CH}_{3}\right), 21.1\left(\mathrm{CH}_{3}\right.$, anti allyl$\left.\mathrm{CH}_{3}\right), 27.4\left(\mathrm{CH}_{3}, \mathrm{~S}-\mathrm{CH}_{3}\right), 39.6\left(\mathrm{CH}_{3}, \mathrm{~N}-\mathrm{CH}_{3}\right), 50.4\left(\mathrm{CH}_{2}\right.$, allyl trans-S), $55.0\left(\mathrm{CH}_{2}, \mathrm{SCH}_{2}\right), 104.2$ (C, allyl), $110.2(\mathrm{CH}$, central allyl), $121.3(\mathrm{CH}$, $\mathrm{CH}=\mathrm{CH} \mathrm{Im}), 123.6(\mathrm{CH}, \mathrm{CH}=\mathrm{CH} \mathrm{Im}), 178.4(\mathrm{C}, \mathrm{NCN})$.

IR ( $\mathrm{KBr}$ pellet, $\mathrm{cm}^{-1}$ ): 1091 (ClO stretching), 623 (ClO bending).

\subsection{2. $\left[\mathrm{Pd}\left(\eta^{3}-1,1-\mathrm{Me}_{2}\right.\right.$ allyl)(NMesityl, $\left.\left.\mathrm{NCH}_{2} \mathrm{SMe}-\mathrm{NHC}\right)\right] \mathrm{ClO}_{4}$ (5b)}

Whitish solid. Yield $79 \%$.

${ }^{1} \mathrm{H}$ NMR $\left(\mathrm{CDCl}_{3}, T=298 \mathrm{~K}, \mathrm{ppm}\right) \delta: 1.35\left(\mathrm{~s}, 3 \mathrm{H}, \mathrm{CH}_{3}\right.$ anti allyl$\left.\mathrm{CH}_{3}\right), 1.94$ (s, 3H, o-aryl- $\left.\mathrm{CH}_{3}\right), 1.97\left(\mathrm{~s}, 3 \mathrm{H}, \mathrm{CH}_{3}\right.$ syn allyl- $\left.\mathrm{CH}_{3}\right) ; 2.02(\mathrm{~s}$, $3 \mathrm{H}, o$-aryl- $\mathrm{CH}_{3}$ ), 2.29 (dd, $J=13.2,3.1 \mathrm{~Hz}, 1 \mathrm{H}$, anti allyl-H), 2.40 (s, $3 \mathrm{H}, p$-aryl- $\mathrm{CH}_{3}$ ), 2.60 (s, 3H, S-CH 3 ), 2.67 (dd, $J=7.4,3.2 \mathrm{~Hz}, 1 \mathrm{H}$, syn allyl), 4.87 (dd, $J=13.2,7.4 \mathrm{~Hz} 1 \mathrm{H}$, central-allyl-H), 5.53 (broad AB system, $2 \mathrm{H}, \mathrm{CH}_{2} \mathrm{~S}$ ), 7.03 (s, $2 \mathrm{H}, m$-aryl-H), $7.06(\mathrm{~d}, J=1.9 \mathrm{~Hz}, 1 \mathrm{H}$, $\mathrm{CH}=\mathrm{CH} \mathrm{Im}), 7.87(\mathrm{~d}, J=1.9 \mathrm{~Hz}, 1 \mathrm{H}, \mathrm{CH}=\mathrm{CH} \mathrm{Im})$.

${ }^{13} \mathrm{C}\left\{{ }^{1} \mathrm{H}\right\} \mathrm{NMR}\left(\mathrm{CDCl}_{3}, T=298 \mathrm{~K}, \mathrm{ppm}\right) \delta: 17.5\left(\mathrm{CH}_{3}, 0\right.$-mesityl- $\left.\mathrm{CH}_{3}\right)$, $17.6\left(\mathrm{CH}_{3}\right.$, o-mesityl- $\left.\mathrm{CH}_{3}\right), 20.1\left(\mathrm{CH}_{3}, \mathrm{~S}-\mathrm{CH}_{3}\right), 20.7\left(\mathrm{CH}_{3}\right.$, anti allyl$\left.\mathrm{CH}_{3}\right), 21.1\left(\mathrm{CH}_{3}, p\right.$-mesityl- $\left.\mathrm{CH}_{3}\right), 27.3\left(\mathrm{CH}_{3}\right.$, syn allyl- $\left.\mathrm{CH}_{3}\right), 50.7\left(\mathrm{CH}_{2}\right.$, allyl), $55.6\left(\mathrm{CH}_{2}, \mathrm{SCH}_{2}\right), 102.9$ (C, allyl), $110.6(\mathrm{CH}$, central allyl), 122.1 $(\mathrm{CH}, \mathrm{CH}=\mathrm{CH}$ Im), $123.2(\mathrm{CH}, \mathrm{CH}=\mathrm{CH} \mathrm{Im}), 129.0(\mathrm{CH}, m$-mesityl- $\mathrm{CH})$;
129.1 (CH, m-mesityl-CH); 134.7 (C, o-mesityl-C); 134.9 (C, o-mesitylC); 136.7(C, i-mesityl-C); 139.8 (C, p-mesityl-C); 189.9 (C, NCN).

IR ( $\mathrm{KBr}$ pellet, $\mathrm{cm}^{-1}$ ): 1089 (ClO stretching), 623 (ClO bending).

\subsection{3. $\left[\mathrm{Pd}\left(\eta^{3}-1,1-\mathrm{Me}_{2}\right.\right.$ allyl)(Ndi-i-propylphenyl, $\left.\left.\mathrm{NCH}_{2} \mathrm{SMe}-\mathrm{NHC}\right)\right]$}

$\mathrm{ClO}_{4}(5 \mathrm{c})$

White solid. Yield 61\%.

${ }^{1} \mathrm{H}$ NMR $\left(\mathrm{CDCl}_{3}, T=298 \mathrm{~K}, \mathrm{ppm}\right) \delta: 1.11\left(\mathrm{~d}, J=6.9 \mathrm{~Hz}, 3 \mathrm{H},{ }^{\mathrm{i}} \mathrm{Pr}-\right.$ $\left.\mathrm{CH}_{3}\right), 1.15\left(\mathrm{~d}, J=6.9 \mathrm{~Hz}, 3 \mathrm{H},{ }^{\mathrm{i}} \mathrm{Pr}-\mathrm{CH}_{3}\right), 1.17\left(\mathrm{~d}, J=6.9 \mathrm{~Hz}, 3 \mathrm{H},{ }^{\mathrm{i}} \mathrm{Pr}-\mathrm{CH}_{3}\right)$, $1.19\left(\mathrm{~d}, J=6.9 \mathrm{~Hz}, 3 \mathrm{H},{ }^{\mathrm{i}} \mathrm{Pr}-\mathrm{CH}_{3}\right), 2.23-2.34(\mathrm{~m}, 2 \mathrm{H}$, anti allyl-H and ${ }^{\mathrm{i}} \mathrm{Pr}-\mathrm{CH}$ ), 2.48 (sept, $\left.1 \mathrm{H}, J=6.9 \mathrm{~Hz},{ }^{\mathrm{i}} \mathrm{Pr}-\mathrm{CH}\right), 2.58$ (s, 3H, S-CH $\mathrm{CH}_{3}, 2.58$ (bd, partially obscured, $1 \mathrm{H}$, syn allyl-H), $5.12(\mathrm{dd}, J=13.4,7.7 \mathrm{~Hz}, 1 \mathrm{H}$, central-allyl-H), 5.57 (bs, $2 \mathrm{H}, \mathrm{CH}_{2} \mathrm{~S}$ ), $7.13(\mathrm{~d}, J=1.9 \mathrm{~Hz}, 1 \mathrm{H}, \mathrm{CH}=\mathrm{CH}$ Im), $7.32(\mathrm{~d}, \mathrm{~J}=7.8 \mathrm{~Hz}, 2 \mathrm{H}, m$-aryl-H), $7.56(\mathrm{t}, J=7.8 \mathrm{~Hz}, 1 \mathrm{H}, p$-aryl$\mathrm{H}), 7.92(\mathrm{~d}, \mathrm{~J}=1.9 \mathrm{~Hz}, 1 \mathrm{H}, \mathrm{CH}=\mathrm{CH} \mathrm{Im})$.

${ }^{13} \mathrm{C}\left\{{ }^{1} \mathrm{H}\right\} \mathrm{NMR}\left(\mathrm{CDCl}_{3}, T=298 \mathrm{~K}, \mathrm{ppm}\right) \delta: 20.1\left(\mathrm{CH}_{3}, \mathrm{~S}-\mathrm{CH}_{3}\right), 20.1\left(\mathrm{CH}_{3}\right.$, $\left.\mathrm{S}-\mathrm{CH}_{3}\right), 21.0\left(\mathrm{CH}_{3}\right.$, anti allyl- $\left.\mathrm{CH}_{3}\right), 23.4\left(\mathrm{CH}_{3},{ }^{\mathrm{i}} \mathrm{Pr}-\mathrm{CH}_{3}\right), 23.6\left(\mathrm{CH}_{3},{ }^{\mathrm{i}} \mathrm{Pr}-\right.$ $\left.\mathrm{CH}_{3}\right), 24.4\left(\mathrm{CH}_{3},{ }^{\mathrm{i}} \mathrm{Pr}-\mathrm{CH}_{3}\right), 24.6\left(\mathrm{CH}_{3},{ }^{\mathrm{i}} \mathrm{Pr}-\mathrm{CH}_{3}\right), 21.0\left(\mathrm{CH}_{3}\right.$, syn allyl- $\left.\mathrm{CH}_{3}\right)$, $28.2\left(\mathrm{CH},{ }^{\mathrm{i}} \mathrm{Pr}-\mathrm{CH}\right), 28.3\left(\mathrm{CH}_{3},{ }^{\mathrm{i}} \mathrm{Pr}-\mathrm{CH}_{3}\right), 52.3\left(\mathrm{CH}_{2}\right.$, allyl $), 55.6\left(\mathrm{CH}_{2}, \mathrm{SCH}_{2}\right)$, 102.7 (C. allyl), $110.4(\mathrm{CH}$, central-allyl), $121.9(\mathrm{CH}, \mathrm{CH}=\mathrm{CH} \mathrm{Im}), 123.9$ $(\mathrm{CH}, m$-aryl-CH); $124.0(\mathrm{CH}, m$-aryl-CH $) ; 124.4(\mathrm{CH}, \mathrm{CH}=\mathrm{CH}$ Im), 130.6 (CH, p-aryl-CH); 136.6 (C, i-aryl-C); 145.5 (C, o-aryl-C); 180.8 (C, NCN). IR ( $\mathrm{KBr}$ pellet, $\mathrm{cm}^{-1}$ ): 1086 (ClO stretching), 621 (ClO bending).

\subsection{4. $\left[\mathrm{Pd}\left(\eta^{3}-1,1-\mathrm{Me}_{2}\right.\right.$ allyl $\left.)\left(\mathrm{NMe}, \mathrm{NCH}_{2} \mathrm{SPh}-\mathrm{NHC}\right)\right] \mathrm{ClO}_{4}(\mathbf{5 d})$}

White solid. Yield $80 \%$.

${ }^{1} \mathrm{H}$ NMR $\left(\mathrm{CD}_{2} \mathrm{Cl}_{2}, T=298 \mathrm{~K}, \mathrm{ppm}\right) \delta: 1.24$ (s, 3H, anti allyl-CH3), 1.89 (s, 3H, syn allyl- $\left.\mathrm{CH}_{3}\right), 3.32$ (dd, $J=12.9,2.2 \mathrm{~Hz}, 1 \mathrm{H}$, anti allyl-H), $3.93\left(\mathrm{~s}, 3 \mathrm{H}, \mathrm{NCH}_{3}\right), 4.28$ (dd, $J=7.5,2.4 \mathrm{~Hz}, 1 \mathrm{H}$, syn allyl-H), 5.20 (dd, $J=12.9,7.5 \mathrm{~Hz}, 1 \mathrm{H}$, central-allyl-H), 5.42, 5.47 (AB system, $\left.J=13.4 \mathrm{~Hz}, 2 \mathrm{H}, \mathrm{CH}_{2} \mathrm{~S}\right), 7.26(\mathrm{~d}, J=1.9 \mathrm{~Hz}, 1 \mathrm{H}, \mathrm{CH}=\mathrm{CH} \mathrm{Im}), 7.46(\mathrm{~d}$, $J=1.9 \mathrm{~Hz}, 1 \mathrm{H}, \mathrm{CH}=\mathrm{CH} \mathrm{Im}), 7.44-7.56(\mathrm{~m}, 5 \mathrm{H}, \mathrm{SPh}-\mathrm{H})$.

${ }^{13} \mathrm{C}\left\{{ }^{1} \mathrm{H}\right\}$ NMR $\left(\mathrm{CDCl}_{3}, T=298 \mathrm{~K}, \mathrm{ppm}\right) \delta: 20.8\left(\mathrm{CH}_{3}\right.$ anti allyl$\left.\mathrm{CH}_{3}\right), 27.2\left(\mathrm{CH}_{3}\right.$ syn allyl- $\left.\mathrm{CH}_{3}\right), 39.7\left(\mathrm{CH}_{3} \mathrm{CH}_{3}-\mathrm{NHC}\right), 51.4\left(\mathrm{CH}_{2}\right.$ allyl $)$, $58.8\left(\mathrm{CH}_{2}, \mathrm{CH}_{2}-\mathrm{SPh}\right), 105.8$ (C allyl), 110.3 ( $\mathrm{CH}$ allyl), $120.8(\mathrm{CH}, \mathrm{CH}=$ $\mathrm{CH} \mathrm{Im}), 124.2(\mathrm{CH}, \mathrm{CH}=\mathrm{CH} \mathrm{Im}), 129.6(\mathrm{C}, \mathrm{i}-\mathrm{Ph}), 130.3(\mathrm{C}, o-\mathrm{Ph}), 131.0$ (C, $p$-Ph), 131.7 (C, $m$-Ph), 178.4 (C, NHC).

IR ( $\mathrm{KBr}$ pellet, $\mathrm{cm}^{-1}$ ): 1085 (ClO stretching), 625 ( $\mathrm{ClO}$ bending).

\subsection{5. $\left[\mathrm{Pd}\left(\eta^{3}-1,1-\mathrm{Me}_{2}\right.\right.$ allyl $\left.)\left(\mathrm{NMe}, \mathrm{NCH}_{2} \mathrm{Py}-\mathrm{NHC}\right)\right] \mathrm{ClO}_{4}$ (5e)}

White solid. Yield $91 \%$.

${ }^{1} \mathrm{H}$ NMR $\left(\mathrm{CD}_{2} \mathrm{Cl}_{2}, T=298 \mathrm{~K}, \mathrm{ppm}\right) \delta: 1.54\left(\mathrm{~s}, 3 \mathrm{H}\right.$, anti allyl- $\left.\mathrm{CH}_{3}\right)$, $1.79\left(\mathrm{~s}, 3 \mathrm{H}\right.$, syn allyl- $\left.\mathrm{CH}_{3}\right), 2.86(\mathrm{dd}, J=12.4,2.8 \mathrm{~Hz}, 1 \mathrm{H}$, anti allyl-H), $3.65\left(\mathrm{dd}, J=7.4,2.8 \mathrm{~Hz}, 1 \mathrm{H}\right.$, syn allyl-H), 3.80 (s, 3H, $\left.\mathrm{NCH}_{3}\right), 5.71$ (dd, $J=12.4,7.4 \mathrm{~Hz}, 1 \mathrm{H}$, central-allyl-H), 5.30, 5.40 (AB system, $\left.J=14.9 \mathrm{~Hz}, 2 \mathrm{H}, \mathrm{CH}_{2} \mathrm{~N}\right), 7.05(\mathrm{~d}, J=1.9 \mathrm{~Hz}, 1 \mathrm{H}, \mathrm{CH}=\mathrm{CH} \mathrm{Im}), 7.46(\mathrm{~d}$, $J=1.9 \mathrm{~Hz}, 1 \mathrm{H}, \mathrm{CH}=\mathrm{CH} \mathrm{Im}$ ), 7.58 (ddd, $J=7.7,4.5,1.4 \mathrm{~Hz}, 1 \mathrm{H}, 5-\mathrm{Pyr}$ ), 7.89 (d, $J=7.7 \mathrm{~Hz}, 1 \mathrm{H}, 3-\mathrm{Pyr}), 8.04$ (td, $1 \mathrm{H}, J=7.7,1.7 \mathrm{~Hz}, 4$-Pyr), 8.52 (d, $1 \mathrm{H}, J=4.5 \mathrm{~Hz}, 6-\mathrm{Pyr})$.

${ }^{13} \mathrm{C}\left\{{ }^{1} \mathrm{H}\right\}$ NMR $\left(\mathrm{CDCl}_{3}, T=298 \mathrm{~K}, \mathrm{ppm}\right) \delta: 20.6\left(\mathrm{CH}_{3}\right.$ anti allyl$\left.\mathrm{CH}_{3}\right), 25.7\left(\mathrm{CH}_{3}\right.$ syn allyl- $\left.\mathrm{CH}_{3}\right), 38.1\left(\mathrm{CH}_{3} \mathrm{CH}_{3}-\mathrm{Im}\right), 40.0\left(\mathrm{CH}_{2}\right.$ allyl $)$, 54.6 ( $\mathrm{CH}_{2} \mathrm{CH}_{2}$-Py), 104.2 (C allyl), 109.7 ( $\mathrm{CH}$ allyl), 121.7 ( $\mathrm{CH}, \mathrm{CH}=$ $\mathrm{CH} \mathrm{Im}$ ), 123.6 ( $\mathrm{CH}, \mathrm{CH}=\mathrm{CH} \mathrm{Im}), 125.4$ ( $\mathrm{CH}$ 5-Py), 127.0 ( $\mathrm{CH}$ 3-Py), 140.2 (CH 4-Py), 150.3 (CH 6-Py), 154.0 (C 2-Py), 175.2 (C, Im).

IR ( $\mathrm{KBr}$ pellet, $\mathrm{cm}^{-1}$ ): 1093 (ClO stretching), 622 (ClO bending).

\subsection{Synthesis of the palladium allyl bis-carbene complexes (6)}

\subsection{1. $\left[\mathrm{Pd}\left(\eta^{3}\right.\right.$-allyl $)\left(\mathrm{NMe}, \mathrm{NCH}_{2} \mathrm{SMe}-\mathrm{NHC}_{2}\right] \mathrm{ClO}_{4}(\mathbf{6 a})$}

To a solution of $0.0528 \mathrm{~g}(0.155 \mathrm{mmol})$ of the complex $\left[\mathrm{Pd}\left(\eta^{3}-\right.\right.$ allyl)(NMe, $\left.\mathrm{NCH}_{2} \mathrm{SMe}^{-\mathrm{NHC}}\right] \mathrm{ClO}_{4}(\mathbf{4 a})$ in $10 \mathrm{~mL}$ of anhydrous $\mathrm{CH}_{2} \mathrm{Cl}_{2}, 0.051 \mathrm{~g}$ (0.155 mmol) of $\mathrm{AgBr}\left(\mathrm{NMe}, \mathrm{NCH}_{2} \mathrm{SMe}-\mathrm{NHC}\right)$ (2a) dissolved in $10 \mathrm{~mL}$ of $\mathrm{CH}_{2} \mathrm{Cl}_{2}$ was added under inert atmosphere (Ar). The reaction mixture was stirred for $1 \mathrm{~h}$ and the precipitated $\mathrm{AgBr}$ filtered off on a millipore filter. The resulting solution was 
concentrated under vacuum and addition of diethyl ether yielded the precipitation of $0.0682 \mathrm{~g}$ (yield $85 \%$ ) of the title complex as a light brown solid. The complex was filtered off on a Gooch, washed with diethyl ether and dried under high vacuum.

${ }^{1} \mathrm{H}$ NMR $\left(\mathrm{CDCl}_{3}, T=298 \mathrm{~K}, \mathrm{ppm}\right) \delta: 2.03\left(\mathrm{~s}, 6 \mathrm{H}, \mathrm{S}-\mathrm{CH}_{3}\right), 2.80(\mathrm{~d}$, $J=13.4 \mathrm{~Hz}, 2 \mathrm{H}$, anti allyl-H), $3.76\left(\mathrm{~s}, 6 \mathrm{H}, \mathrm{N}-\mathrm{CH}_{3}\right), 3.98(\mathrm{~d}, J=6.4 \mathrm{~Hz}$, $2 \mathrm{H}$, syn allyl-H), $4.92\left(\mathrm{~s}, 4 \mathrm{H}, \mathrm{CH}_{2} \mathrm{~S}\right), 5.44(\mathrm{~m}, 1 \mathrm{H}$, central-allyl-H), 7.16 (d, $J=1.9 \mathrm{~Hz}, 2 \mathrm{H}, \mathrm{CH}=\mathrm{CH} \mathrm{Im}), 7.32(\mathrm{~d}, J=1.9 \mathrm{~Hz}, 2 \mathrm{H}, \mathrm{CH}=\mathrm{CH} \mathrm{Im})$.

${ }^{13} \mathrm{C}\left\{{ }^{1} \mathrm{H}\right\}$ NMR $\left(\mathrm{CDCl}_{3}, T=298 \mathrm{~K}, \mathrm{ppm}\right) \delta: 14.4\left(\mathrm{CH}_{3}, \mathrm{~S}-\mathrm{CH}_{3}\right), 38.5$ $\left(\mathrm{CH}_{3}, \mathrm{~N}-\mathrm{CH}_{3}\right), 53.8\left(\mathrm{CH}_{2}, \mathrm{SCH}_{2}\right), 60.2\left(\mathrm{CH}_{2}\right.$, allyl $), 119.1(\mathrm{CH}$, central allyl), $122.2(\mathrm{CH}, \mathrm{CH}=\mathrm{CH} \mathrm{Im}), 123.6(\mathrm{CH}, \mathrm{CH}=\mathrm{CH} \mathrm{Im}), 176.9(\mathrm{C}$, NCN).

IR (KBr pellet, $\mathrm{cm}^{-1}$ ): 1092 (ClO stretching), 623 ( $\mathrm{ClO}$ bending). Anal. Calcd. for $\mathrm{C}_{15} \mathrm{H}_{25} \mathrm{ClN}_{4} \mathrm{O}_{4} \mathrm{PdS}_{2}$ : C, 33.90; H, 4.74; N, 10.54 . Found: C, 33.79; $\mathrm{H}, 4.63 ; \mathrm{N}, 10.42 \%$.

The following complexes were synthesized by a similar procedure using the appropriate complexes.

\subsection{2. $\left[\mathrm{Pd}\left(\eta^{3}\right.\right.$-allyl $\left.)\left(\mathrm{NMe}, \mathrm{NCH}_{2} \mathrm{SPh}-\mathrm{NHC}\right)_{2}\right] \mathrm{ClO}_{4}(\mathbf{6 d})$}

Whitish solid. Yield $91 \%$.

${ }^{1} \mathrm{H}$ NMR $\left(\mathrm{CD}_{2} \mathrm{Cl}_{2}, T=298 \mathrm{~K}, \mathrm{ppm}\right) \delta: 2.59(\mathrm{~d}, J=13.2 \mathrm{~Hz}, 2 \mathrm{H}$, anti allyl-H), 3.70 (s, 6H, NCH 3 ), 3.78 (d, J = 7.5 Hz, 2H, syn allyl-H), 5.16 $\left(\mathrm{s}, 4 \mathrm{H}, \mathrm{CH}_{2} \mathrm{~S}\right), 5.22(\mathrm{~m}, 1 \mathrm{H}$, central-allyl-H), $7.00(\mathrm{~d}, J=1.9 \mathrm{~Hz}, 2 \mathrm{H}$, $\mathrm{CH}=\mathrm{CH} \mathrm{Im}), 7.10(\mathrm{~d}, J=1.9 \mathrm{~Hz}, 2 \mathrm{H}, \mathrm{CH}=\mathrm{CH} \mathrm{Im}), 7.43-7.44(\mathrm{~m}, 10 \mathrm{H}$, $\mathrm{SPh}-\mathrm{H})$.

${ }^{13} \mathrm{C}\left\{{ }^{1} \mathrm{H}\right\} \mathrm{NMR}\left(\mathrm{CDCl}_{3}, \mathrm{~T}=298 \mathrm{~K}, \mathrm{ppm}\right) \delta: 38.5\left(\mathrm{CH}_{3} \mathrm{CH}_{3}-\mathrm{Im}\right), 55.9$ ( $\mathrm{CH}_{2} \mathrm{CH}_{2}-\mathrm{SPh}$ ), 60.3 ( $\mathrm{CH}_{2}$ allyl), 119.2 ( $\mathrm{CH}$ allyl), $121.9(\mathrm{CH}, \mathrm{CH}=\mathrm{CH}$ Im), 123.6 ( $\mathrm{CH}, \mathrm{CH}=\mathrm{CH}$ Im), 128.9 (C, $p$-Ph), 129.5 (C, o-Ph), 132.0 (C, $i-\mathrm{Ph}), 133.2$ (C, $m-\mathrm{Ph}), 176.8$ (C, Im).

IR ( $\mathrm{KBr}$ pellet, $\mathrm{cm}^{-1}$ ): 1093 (ClO stretching), 622 (ClO bending).

Anal. Calcd. for $\mathrm{C}_{25} \mathrm{H}_{29} \mathrm{ClN}_{4} \mathrm{O}_{4} \mathrm{PdS}_{2}$ : C, 45.81; $\mathrm{H}, 4.46 ; \mathrm{N}, 8.55$. Found: C, 45.72; $\mathrm{H}, 4.35$; N, 8.63\%.

\subsection{3. $\left[\mathrm{Pd}\left(\eta^{3}\right.\right.$-allyl $\left.)\left(\mathrm{NMe}, \mathrm{NCH}_{2} \mathrm{Py}-\mathrm{NHC}\right)_{2}\right] \mathrm{ClO}_{4}(\mathbf{6 e})$}

Whitish solid. Yield $89 \%$.

${ }^{1} \mathrm{H}$ NMR $\left(\mathrm{CD}_{2} \mathrm{Cl}_{2}, T=298 \mathrm{~K}, \mathrm{ppm}\right) \delta: 2.55(\mathrm{~d}, J=13.2 \mathrm{~Hz}, 2 \mathrm{H}$, anti allyl-H), $3.67\left(\mathrm{~s}, 6 \mathrm{H}, \mathrm{NCH}_{3}\right), 3.80(\mathrm{~d}, J=6.8 \mathrm{~Hz}, 2 \mathrm{H}$, syn allyl-H), 5.21 (m, $1 \mathrm{H}$, central-allyl-H), $5.25\left(\mathrm{~s}, 4 \mathrm{H}, \mathrm{CH}_{2} \mathrm{~N}\right), 6.88(\mathrm{~d}, J=7.7 \mathrm{~Hz}, 2 \mathrm{H}, 3-$ Pyr); $7.04(\mathrm{~d}, J=1.9 \mathrm{~Hz}, 2 \mathrm{H}, \mathrm{CH}=\mathrm{CH} \mathrm{Im}), 7.07$ (d, $J=1.9 \mathrm{~Hz}, 2 \mathrm{H}, \mathrm{CH}=$ CH Im), 7.05 (dd, $J=7.7,4.1 \mathrm{~Hz}, 2 \mathrm{H}, 5-\mathrm{Pyr}$ ), 7.65 (td, 2H, $J=7.6$, $1.7 \mathrm{~Hz}, 4-\mathrm{Pyr}), 8.50$ (d, 2H, $J=4.1 \mathrm{~Hz}, 6-\mathrm{Pyr}$ ).

${ }^{13} \mathrm{C}\left\{{ }^{1} \mathrm{H}\right\} \mathrm{NMR}\left(\mathrm{CDCl}_{3}, \mathrm{~T}=298 \mathrm{~K}, \mathrm{ppm}\right) \delta: 38.2\left(\mathrm{CH}_{3} \mathrm{CH}_{3}-\mathrm{Im}\right), 55.7$ ( $\mathrm{CH}_{2} \mathrm{CH}_{2}$-Py), 60.0 ( $\mathrm{CH}_{2}$ allyl), 119.0 ( $\mathrm{CH}$ allyl), $121.3(\mathrm{CH}, \mathrm{CH}=\mathrm{CH}$ Im), 122.6 (CH 5-Py), 122.8 (CH 3-Py), 123.6 (CH, CH=CH Im), 137.3 (CH 4-Py), 149.3 (CH 6-Py), 155.4 (C 2-Py), 177.3 (C, Im).

IR ( $\mathrm{KBr}$ pellet, $\left.\mathrm{cm}^{-1}\right) \nu$ : 1593 (CN Py stretching), 1093 (ClO stretching), 622 ( $\mathrm{ClO}$ bending).

Anal. Calcd. for $\mathrm{C}_{23} \mathrm{H}_{27} \mathrm{ClN}_{6} \mathrm{O}_{4} \mathrm{Pd}$ : C, 46.56; $\mathrm{H}, 4.59 ; \mathrm{N}, 14.16$. Found: C, 46.48; $\mathrm{H}, 4.70 ; \mathrm{N}, 14.25 \%$.

\subsection{4. $\left[\mathrm{Pd}\left(\eta^{3}\right.\right.$-allyl $\left.)\left(\mathrm{NMe}, \mathrm{NCH}_{2} \mathrm{Ph}-\mathrm{NHC}\right)_{2}\right] \mathrm{BF}_{4}(\mathbf{6 f})$}

To a solution of $0.1 \mathrm{~g}(0.28 \mathrm{mmol})$ of the complex $\left[\mathrm{Pd}\left(\eta^{3}-\right.\right.$ allyl)(NMe, $\left.\mathrm{NCH}_{2} \mathrm{Ph}-\mathrm{NHC}\right) \mathrm{Cl}$ ] $(3 \mathrm{~h})$ in $8 \mathrm{~mL}$ of anhydrous $\mathrm{CH}_{2} \mathrm{Cl}_{2}$, $0.101 \mathrm{~g}(0.28 \mathrm{mmol})$ of $\mathrm{AgBr}\left(\mathrm{NMe}, \mathrm{NCH}_{2} \mathrm{Ph}-\mathrm{NHC}\right)$ (2f) dissolved in $7 \mathrm{~mL}$ of anhydrous $\mathrm{CH}_{2} \mathrm{Cl}_{2}$ were added under inert atmosphere (Ar). To the resulting solution $0.055 \mathrm{~g}(0.28 \mathrm{mmol})$ of $\mathrm{AgBF}_{4}$ were added and the resulting mixture was vigorously stirred for $30 \mathrm{~min}$ and the precipitated $\mathrm{AgBr}$ filtered off on a millipore filter. The clear solution was concentrated under vacuum to the final volume of $2 \mathrm{~mL}$ and treated with diethyl ether $(10 \mathrm{~mL})$. The precipitated oil was separated by decantation and dried under vacuum. $0.155 \mathrm{~g}$ (yield 95\%) of the title complex was isolated as a whitish solid.

${ }^{1} \mathrm{H}$ NMR $\left(\mathrm{CDCl}_{3}, T=298 \mathrm{~K}, \mathrm{ppm}\right) \delta: 2.64(\mathrm{~d}, J=13.3 \mathrm{~Hz}, 2 \mathrm{H}$, anti allyl-H), $3.71\left(\mathrm{~s}, 6 \mathrm{H}, \mathrm{N}-\mathrm{CH}_{3}\right), 3.85$ (d, $J=7.4 \mathrm{~Hz}, 2 \mathrm{H}$, syn allyl-H), 5.12 $\left(\mathrm{s}, 4 \mathrm{H}, \mathrm{CH}_{2} \mathrm{~N}\right), 5.27(\mathrm{~m}, 1 \mathrm{H}$, central allyl-H), 6.88-6.92 $(\mathrm{m}, 12 \mathrm{H}, \mathrm{CH}=$
$\mathrm{CH}$ Im, Ph-H), $6.87(\mathrm{~d}, J=1.9 \mathrm{~Hz}, 2 \mathrm{H}, \mathrm{CH}=\mathrm{CH} \mathrm{Im}), 7.28-7.32(\mathrm{~m}, 6 \mathrm{H}$, $\mathrm{Ph}-\mathrm{H}) .{ }^{13} \mathrm{C}\left\{{ }^{1} \mathrm{H}\right\}$ NMR $\left(\mathrm{CDCl}_{3}, \mathrm{~T}=298 \mathrm{~K}, \mathrm{ppm}\right) .{ }^{13} \mathrm{C}\left\{{ }^{1} \mathrm{H}\right\} \mathrm{NMR}\left(\mathrm{CDCl}_{3}\right.$, $T=298 \mathrm{~K}, \mathrm{ppm}) \delta: 38.1\left(\mathrm{CH}_{3}, \mathrm{~N}-\mathrm{CH}_{3}\right), 54.0\left(\mathrm{CH}_{2}, \mathrm{Ph}-\mathrm{CH}_{2}\right), 60.1\left(\mathrm{CH}_{2}\right.$, $\mathrm{CH}_{2}$-allyl), 119.1 ( $\mathrm{CH}, \mathrm{CH}$-allyl), $122.2(\mathrm{CH}, \mathrm{CH}=\mathrm{CH} \mathrm{Im}), 123.9(\mathrm{CH}$, $\mathrm{CH}=\mathrm{CH} \mathrm{Im}), 126.4(\mathrm{CH}, m-\mathrm{Ph}) ; 128.0(\mathrm{CH}, p-\mathrm{Ph}) ; 128.8(\mathrm{CH}, o-\mathrm{Ph})$; 135.7 (C, $i-\mathrm{Ph}) ; 176.8$ (C, NCN).

Anal. Calcd. for $\mathrm{C}_{25} \mathrm{H}_{29} \mathrm{BF}_{4} \mathrm{~N}_{4} \mathrm{Pd}$ : C, 51.88; $\mathrm{H}, 5.05 ; \mathrm{N}, 9.68$. Found: C, 51.79 ; H, 4.95; N, 9.46\%.

The following complex was synthesized by a similar procedure.

\subsection{5. $\left[\mathrm{Pd}\left(\eta^{3}\right.\right.$-allyl $\left.)\left(\mathrm{NMe}, \mathrm{NCH}_{2} \mathrm{Tol}-\mathrm{NHC}\right)_{2}\right] \mathrm{BF}_{4}(\mathbf{6 g})$}

Whitish solid. Yield $89 \%$.

${ }^{1} \mathrm{H}$ NMR $\left(\mathrm{CDCl}_{3}, T=298 \mathrm{~K}, \mathrm{ppm}\right) \delta: 2.29\left(\mathrm{~s}, 6 \mathrm{H}\right.$, tolyl- $\left.\mathrm{CH}_{3}\right), 2.62$ (d, $J=13.3 \mathrm{~Hz}, 2 \mathrm{H}$, anti allyl-H), $3.67\left(\mathrm{~s}, 6 \mathrm{H}, \mathrm{N}-\mathrm{CH}_{3}\right), 3.87$ (d, $J=7.3 \mathrm{~Hz}, 2 \mathrm{H}$, syn allyl-H), $5.04\left(\mathrm{~s}, 4 \mathrm{H}, \mathrm{CH}_{2} \mathrm{~N}\right), 5.26(\mathrm{~m}, 1 \mathrm{H}$, central allyl-H), 6.79 (d, $J=7.9 \mathrm{~Hz}, 2 \mathrm{H}$, tolyl-H), 6.87 (d, $J=1.8 \mathrm{~Hz}, 2 \mathrm{H}$, $\mathrm{CH}=\mathrm{CH} \mathrm{Im}), 7.06-7.10\left(\mathrm{~m}, 6 \mathrm{H}, \mathrm{CH}=\mathrm{CH}\right.$ Im, tolyl-H). ${ }^{13} \mathrm{C}\left\{{ }^{1} \mathrm{H}\right\} \mathrm{NMR}$ $\left(\mathrm{CDCl}_{3}, T=298 \mathrm{~K}, \mathrm{ppm}\right) \delta: 21.0\left(\mathrm{CH}_{3}, \mathrm{Ph}-\mathrm{CH}_{3}\right), 38.1\left(\mathrm{CH}_{3}, \mathrm{~N}-\mathrm{CH}_{3}\right)$, $53.9\left(\mathrm{CH}_{2}, \mathrm{Ph}-\mathrm{CH}_{2}\right), 60.0\left(\mathrm{CH}_{2}, \mathrm{CH}_{2}\right.$-allyl $), 119.1(\mathrm{CH}, \mathrm{CH}$-allyl), $122.1(\mathrm{CH}, \mathrm{CH}=\mathrm{CH} \mathrm{Im}), 123.9(\mathrm{CH}, \mathrm{CH}=\mathrm{CH} \mathrm{Im}), 126.5(\mathrm{CH}, m-$ $\mathrm{Ph}) ; 129.5$ (CH, o-Ph); 132.6 (C, $i-\mathrm{Ph}) ; 137.9$ (C, p-Ph); 176.4 (C, NCN).

Anal. Calcd. for $\mathrm{C}_{27} \mathrm{H}_{33} \mathrm{BF}_{4} \mathrm{~N}_{4} \mathrm{Pd}$ : C, 53.44; $\mathrm{H}, 5.48 ; \mathrm{N}, 9.23$. Found: C, 53.37; H, 5.36; N, 9.03\%.

The complexes 7, 8 and 9 cannot be separated in their pure form and were identified in solution from their relevant ${ }^{1} \mathrm{H}$ NMR signals among the well-known peaks belonging to the symmetric bis-carbene complexes concurring to their formation.

\subsection{6. $\left[\mathrm{Pd}\left(\eta^{3} \text {-allyl }\right)\left(\mathrm{NMe}, \mathrm{NCH}_{2} \mathrm{SMe}-\mathrm{NHC}\right)\left(\mathrm{NMe}, \mathrm{NCH}_{2} \mathrm{Py}-\mathrm{NHC}\right)\right]^{+}$(7)}

${ }^{1} \mathrm{H}$ NMR $\left(\mathrm{CD}_{2} \mathrm{Cl}_{3}, T=298 \mathrm{~K}, \mathrm{ppm}\right) \delta: 2.01\left(\mathrm{~s}, 3 \mathrm{H}, \mathrm{S}-\mathrm{CH}_{3}\right), 2.66(\mathrm{~d}$, $J=12.4 \mathrm{~Hz}, 1 \mathrm{H}$, anti allyl-H), $2.74(\mathrm{~d}, J=13.6 \mathrm{~Hz}, 1 \mathrm{H}$, anti allyl-H), $3.65\left(\mathrm{~s}, 3 \mathrm{H}, \mathrm{N}-\mathrm{CH}_{3}\right), \delta: 3.78\left(\mathrm{~s}, 3 \mathrm{H}, \mathrm{NCH}_{3}\right), 3.90(\mathrm{~d}, J=7.0 \mathrm{~Hz}, 1 \mathrm{H}$, syn allyl-H), 3.95 (d, $J=7.5 \mathrm{~Hz}, 1 \mathrm{H}$, syn allyl-H), $4.88\left(\mathrm{~s}, 2 \mathrm{H}, \mathrm{CH}_{2} \mathrm{~S}\right)$, 5.28 (s, 2H, CH${ }_{2} \mathrm{~N}$ ), 6.86 (d, $\left.J=7.0 \mathrm{~Hz}, 1 \mathrm{H}, 3-\mathrm{Pyr}\right), 6.98$ (d, $J=1.7 \mathrm{~Hz}$, $1 \mathrm{H}, \mathrm{CH}=\mathrm{CH} \mathrm{Im}), 7.05(\mathrm{dd}, J=7.7,4.1 \mathrm{~Hz}, 1 \mathrm{H}, 5-\mathrm{Pyr}), 7.17$ (d, $J=1.9 \mathrm{~Hz}, 1 \mathrm{H}, \mathrm{CH}=\mathrm{CH} \mathrm{Im}), 7.21(\mathrm{~d}, J=2.1 \mathrm{~Hz}, 1 \mathrm{H}, \mathrm{CH}=\mathrm{CH} \mathrm{Im}), 7.67$ (td, $1 \mathrm{H}, J=7.6,1.6 \mathrm{~Hz}, 4-\mathrm{Pyr}), 8.52$ (d, 1H, $J=5.2 \mathrm{~Hz}, 6-\mathrm{Pyr}$ ).

\subsection{7. $\left[\mathrm{Pd}\left(\eta^{3} \text {-allyl }\right)\left(\mathrm{NMe}, \mathrm{NCH}_{2} \mathrm{SMe}-\mathrm{NHC}\right)\left(\mathrm{NMe}, \mathrm{NCH}_{2} \mathrm{Ph}-\mathrm{NHC}\right)\right]^{+}(\boldsymbol{8})$}

${ }^{1} \mathrm{H}$ NMR $\left(\mathrm{CD}_{2} \mathrm{Cl}_{2}, T=298 \mathrm{~K}, \mathrm{ppm}\right) \delta: 2.03\left(\mathrm{~s}, 3 \mathrm{H}, \mathrm{S}-\mathrm{CH}_{3}\right), 2.73(\mathrm{~d}$, $J=13.4 \mathrm{~Hz}, 1 \mathrm{H}$, anti allyl-H), $2.76(\mathrm{~d}, J=13.6 \mathrm{~Hz}, 1 \mathrm{H}$, anti allyl- $\mathrm{H})$, $3.62\left(\mathrm{~s}, 3 \mathrm{H}, \mathrm{N}-\mathrm{CH}_{3}\right), 3.79\left(\mathrm{~s}, 3 \mathrm{H}, \mathrm{N}-\mathrm{CH}_{3}\right), 3.98(\mathrm{~d}, J=7.2 \mathrm{~Hz}, 2 \mathrm{H}$, syn allyl- $\mathrm{H}), 4.85\left(\mathrm{~s}, 2 \mathrm{H}, \mathrm{CH}_{2} \mathrm{~S}\right), 5.21\left(\mathrm{~s}, 2 \mathrm{H}, \mathrm{CH}_{2} \mathrm{~N}\right), 6.90-6.94(\mathrm{~m}, 2 \mathrm{H}$, $\mathrm{Ph}-\mathrm{H}), 7.02$ (d, $J=1.8 \mathrm{~Hz}, 1 \mathrm{H}, \mathrm{CH}=\mathrm{CH} \mathrm{Im}), 7.03(\mathrm{~d}, J=1.7 \mathrm{~Hz}, 1 \mathrm{H}$, $\mathrm{CH}=\mathrm{CH} \operatorname{Im}), 7.20(\mathrm{~d}, J=1.9 \mathrm{~Hz}, 1 \mathrm{H}, \mathrm{CH}=\mathrm{CH} \mathrm{Im}), 7.24(\mathrm{~d}, J=1.9 \mathrm{~Hz}$, $2 \mathrm{H}, \mathrm{CH}=\mathrm{CH}$ Im), 7.32-7.35 (m, 3H, Ph-H).

\subsection{8. $\left[\mathrm{Pd}\left(\eta^{3} \text {-allyl }\right)\left(\mathrm{NMe}, \mathrm{NCH}_{2} \mathrm{Ph}-\mathrm{NHC}\right)\left(\mathrm{NMe}, \mathrm{NCH}_{2} \mathrm{Tol}-\mathrm{NHC}\right)\right]^{+}(\mathbf{9})$}

${ }^{1} \mathrm{H}$ NMR $\left(\mathrm{CD}_{2} \mathrm{Cl}_{2}, T=298 \mathrm{~K}, \mathrm{ppm}\right) \delta: 2.34\left(\mathrm{~s}, 3 \mathrm{H}\right.$, tolyl- $\left.\mathrm{CH}_{3}\right), 2.66$ (d, $J=12.8 \mathrm{~Hz}, 2 \mathrm{H}$, anti allyl-H), $3.68\left(\mathrm{~s}, 3 \mathrm{H}, \mathrm{N}-\mathrm{CH}_{3}\right), 3.70(\mathrm{~s}, 3 \mathrm{H}, \mathrm{N}-$ $\left.\mathrm{CH}_{3}\right), 3.89-3.96\left(\mathrm{~m}, 2 \mathrm{H}\right.$, syn allyl-H), $5.06\left(\mathrm{~s}, 2 \mathrm{H}, \mathrm{CH}_{2} \mathrm{~N}\right), 5.12(\mathrm{~s}, 2 \mathrm{H}$, $\left.\mathrm{CH}_{2} \mathrm{~N}\right), 5.26(\mathrm{~m}, 1 \mathrm{H}$, central allyl-H), $6.83(\mathrm{~d}, J=7.2 \mathrm{~Hz}$, tolyl-H), 6.90-6.98 (m, 2H, Ph-H), 7.07-7.17 (m, CH=CH Im), 7.30-7.37 $(\mathrm{m}, 3 \mathrm{H}, \mathrm{Ph}-\mathrm{H})$.

\subsection{Crystal structure determinations}

The crystal data of compounds $\mathbf{5 b}$ and $\mathbf{6 d}$ were collected at room temperature using a Nonius Kappa CCD diffractometer with graphite monochromated Mo-K $\alpha$ radiation. The data sets were integrated with the Denzo-SMN package [29] and corrected for Lorentz, polarization and absorption effects (SORTAV) [30]. The structures were solved by direct methods using SIR97 [31] system of programs and refined using full-matrix least-squares with all 
non-hydrogen atoms anisotropically and hydrogens included on calculated positions, riding on their carrier atoms.

The asymmetric unit of $\mathbf{5 b}$ contains two independent ionic couples with one cationic complex displaying a disordered 1,1dimethyl substituted allyl group whose central C2 atom and both methyl groups were refined over two positions with occupancies of 0.73 and 0.27 , respectively.

All calculations were performed using SHELXL-97 [32] and PARST [33] implemented in WINGX [34] system of programs. The crystal data are given in Table $1 \mathrm{SI}$.

\section{Appendix A. Supplementary material}

CCDC 894208 and 894209 contain the supplementary crystallographic data for this paper. These data can be obtained free of charge from The Cambridge Crystallographic Data Centre via www. ccdc.cam.ac.uk/data_request/cif.

\section{Appendix B. Supplementary data}

Supplementary data related to this article can be found at http:// dx.doi.org/10.1016/j.jorganchem.2013.01.016.

\section{References}

[1] (a) W.A. Hermann, Angew. Chem. Int. Ed. 41 (2002) 1290;

(b) C.M. Crudden, D.P. Allen, Coord. Chem. Rev. 248 (2004) 2247;

(c) N.M. Scott, S.P. Nolan, Eur. J. Inorg. Chem. (2005) 1815;

(d) S. Diez-González, S.P. Nolan, Coord. Chem. Rev. 251 (2007) 874;

(e) E.A.B. Kantchev, C.J. O'Brien, M.G. Organ, Angew. Chem. Int. Ed. 46 (2007) 2768;

(f) F.E. Hahn, M.C. Jahnke, Angew. Chem. Int. Ed. 47 (2008) 3122;

(g) A.T. Normand, K.J. Cavell, Eur. J. Inorg. Chem. (2008) 2781;

(h) F. Boeda, S.P. Nolan, Annu. Rep. Sect. B (Org. Chem.) 104 (2008) 184;

(i) U. Radius, F.M. Bickelhaupt, Coord. Chem. Rev. 253 (2009) 678;

(j) H. Jacobsen, A. Correa, A. Poater, C. Constable, L. Cavallo, Coord. Chem. Rev. (2009) 687.

[2] (a) H.M. Lee, C.-C. Lee, P.-Y. Cheng, Curr. Org. Chem. 11 (2007) 1491;

(b) M.C. Jahnke, T. Pape, F.E. Hahn, Eur. J. Inorg. Chem. (2009) 1960;

(c) D. Meyer, M.A. Taige, A. Zeller, K. Hohlfeld, S. Ahrens, T. Strassner, Organometallics 28 (2009) 2142;

(d) A.R. Chianese, P.T. Bremer, C. Wong, R.J. Reynes, Organometallics 28 (2009) 5244

(e) W.N.O. Wylie, A.J. Lough, R.H. Morris, Organometallics 28 (2009) 6755;

(f) S. Warsink, P. Hauwert, M.A. Siegler, A.L. Spek, C.J. Elsevier, Appl. Organomet. Chem. 23 (2009) 225;

(g) S. Warsink, S.Y.D. Boer, L.M. Jongens, C.-F. Fu, S.-T. Liu, J.-T. Chen, M. Lutz, A.L. Spek, C.J. Elsevier, Dalton Trans. (2009) 7080;

(h) C. Lu, S. Gu, W. Chen, H. Qiu, Dalton Trans. 39 (2010) 4198;

(i) S. Warsink, R.M. Drost, M. Lutz, A.L. Spek, C.J. Elsevier, Organometallics 29 (2010) 3109.

[3] P. Braunstein, F. Naud, Angew. Chem. Int. Ed. 40 (2001) 680.

[4] L. Canovese, G. Chessa, F. Visentin, P. Uguagliati, Coord. Chem. Rev. 248 (2004) 945.

[5] (a) D. Sellmann, W. Prechtel, F. Knoch, M. Moll, Organometallics 11 (1992) 2346;

(b) H. Seo, H.J. Park, B.Y. Kim, J.H. Lee, S.U. Son, Y.K. Chung, Organometallics 22 (2003) 618;

(c) A. Ros, D. Monge, M. Alcarazo, E. Álvarez, J.M. Lassaletta, R.R. Fernàndez, Organometallics 26 (2006) 6039;

(d) S.J. Roseblade, A. Ros, D. Monge, M. Alcarazo, E. Alvarez, J.M. Lassaletta, R. Fernàndez, Organometallics 26 (2007) 2570;

(e) E. Kluser, A. Neels, M. Albrecht, Chem. Commun. (2006) 4495

(f) H.V. Huynh, C.H. Yeo, G.K. Tan, Chem. Commun. (2006) 3833;

(g) J. Wolf, A. Labande, J.-C. Daran, R. Poli, Eur. J. Inorg. Chem. (2007) 5069;

(h) D.S. Mc Guinness, J.A. Suttil, M.G. Gardiner, N.W. Davis, Organometallics 27 (2008) 4238;

(i) C. Gandolfi, M. Heckenroth, A. Neels, G. Laurenczy, M. Albrecht, Organometallics 28 (2009) 5112;

(j) C. Fliedel, G. Schnee, P. Braunstein, Dalton Trans. (2009) 2474;

(k) J. Iglesias, A. Ros, E. Diez, A. Magriz, A. Vazquez, E. Alvarez, R. Fernandez, J.M. Lassaletta, Dalton Trans. (2009) 8485;

(1) S.-T. Liu, C.-I. Lee, C.-F. Fu, C.-H. Chen, Y.-H. Liu, C.J. Elsevier, S.-M. Peng, J.-T. Chen, Organometallics 28 (2009) 6597;

(m) C. Fliedel, A. Sabbatini, P. Braunstein, Dalton Trans. 39 (2010) 8820;

(n) H.V. Huynh, C.H. Yeo, Y.X. Chew, Organometallics 29 (2010) 1479; (o) D. Yuan, H.V. Huynh, Organometallics 29 (2010) 6020;

(p) D. Yuan, H. Tang, L. Xiao, H.V. Huynh, Dalton Trans. 40 (2011) 8788.

[6] N. Marion, S.P. Nolan, Acc. Chem. Res. 41 (2008) 1440.

[7] (a) M.S. Viciu, O. Navarro, R.F. Germaneau, R.A. Kelly, W. Sommer, N. Marion, E.D. Stevens, L. Cavallo, S.P. Nolan, Organometallics 23 (2004) 1629;

(b) K.J. Cavell, D.S. McGuinness, Coord. Chem. Rev. 248 (2004) 671;

(d) S. Roland, M. Audouin, P. Mangeney, Organometallics 23 (2004) 3075;

(e) Y. Ding, R. Goddard, K.-R. Pörschke, Organometallics 24 (2005) 439;

(f) E.S. Chernyshova, R. Goddard, K.-R. Pörschke, Organometallics 26 (2007) 3236;

(g) A.T. Normand, A. Stasch, L.-L. Ooi, K.J. Cavell, Organometallics 26 (2007) 4863;

(h) X. Luan, L. Wu, E. Drinkel, R. Mariz, M. Gatti, R. Dorta, Org. Lett. 12 (2010) 1912;

(i) Y.-X. Jia, D. Katayev, G. Bernadinelli, T.M. Seidel, E.P. Kündig, Chem. Eur. J. 16 (2010) 6300;

(j) M.S. Jeletic, I. Ghiviriga, K.A. Abboud, A.S. Veige, Dalton Trans. 39 (2010) 6392; (k) P.V. Simpson, B.W. Skelton, D.H. Brown, M.V. Baker, Eur. J. Inorg. Chem. (2011) 1937;

(1) J. Wu, N. Hazari, Chem. Commun. 47 (2011) 1069;

(m) J.D. Egbert, A. Chartoire, A.M.Z. Slavin, S.P. Nolan, Organometallics 30 (2011) 4494.

[8] (a) A.A. Danopoulos, N. Tsoureas, S.A. Macgregor, C. Smith, Organometallics 26 (2007) 253;

(b) C.-Y. Wang, Y.-H. Liu, S.-M. Peng, J.-T. Chen, S.-T. Liu, J. Organomet. Chem. 692 (2007) 3976;

(c) N.T. Barczak, R.E. Grote, E. Jarvo, Organometallics 26 (2007) 4863.

[9] (a) D.S. McGuinness, K.J. Cavell, Organometallics 19 (2000) 741;

(b) M. Frøseth, D.S. Netland, K.W. Törnroos, A. Dhindsa, M. Tilset, Dalton Trans. (2005) 1664;

(c) N.D. Clement, K.J. Cavell, L.-L. Ooi, Organometallics 25 (2006) 4155.

[10] (a) D.S. McGuinness, K.J. Cavell, B.W. Skelton, A.H. White, Organometallics 18 (1999) 1596;

(b) J.-Y. Lee, P.-Y. Cheng, Y.-H. Tsai, G.-R. Lin, S.-P. Liu, M.-H. Sie, H.M. Lee, Organometallics 29 (2010) 3901.

[11] (a) E.O. Fischer, H.-J. Beck, Angew. Chem. Int. Ed. Engl. 9 (1970) 72;

(b) E.O. Fischer, H.-J. Beck, C.G. Kreiter, J. Lynch, J. Müller, E. Winkler, Chem. Ber. 105 (1972) 162;

(c) L. Jordi, J.M. Moretó, S. Ricart, J.M. Viñas, M. Mejias, E. Molins, Organometallics 11 (1992) 3507;

(d) S.-T. Liu, T.-Y. Hsieh, G.-H. Lee, S.-M. Peng, Organometallics 17 (1998) 993; (e) R.-Z. Ku, J.-C. Huang, J.-Y. Cho, F.-M. Kiang, K.R. Reddy, Y.-C. Chen, K.-J. Lee, J.-H. Lee, G.-H. Lee, S.-M. Peng, S.-T. Liu, Organometallics 18 (1999) 2145;

(f) J. Barluenga, R. Vicente, L.A. López, E. Rubio, M. Tomás, C. Àlvarez-Rúa, J. Am. Chem. Soc. 126 (2004) 470;

(g) J. Barluenga, R. Vicente, L.A. López, M. Tomás, J. Organomet. Chem. 691 (2006) 5642;

(h) J. Barluenga, R. Vicente, P. Barrio, L.A. López, M. Tomás, J. Am. Chem. Soc. 126 (2004) 5974;

(i) J. Barluenga, R. Vicente, P. Barrio, L.A. López, M. Tomás, J. Borge, J. Am. Chem. Soc. 126 (2004) 14354;

(j) Y.-H. Chang, C.-F. Fu, Y.-H. Liu, S.-M. Peng, J.-T. Chen, S.-T. Liu, Dalton Trans, (2009) 861;

(k) Y.-H. Chang, C.-F. Fu, Y.-H. Liu, S.-M. Peng, C.J. Elsevier, J.-T. Chen, S.-T. Liu, Dalton Trans. (2009) 6991;

(1) J. Barluenga, P. Barrio, L.A. López, M. Tomás, S. García-Granda, C. ÀlvarezRúa, Angew. Chem. Int. Ed. 42 (2003) 3008;

(m) J.C. del Amo, M.J. Mancheño, M. Gómez-Gallego, M.A. Sierra, Organometallics 23 (2004) 5021;

(n) F. Kessler, N. Szesni, C. Maass, C. Hohberger, B. Weibert, H. Fischer, J. Organomet. Chem. 692 (2007) 3005;

(o) M.A. Sierra, M.J. Mancheño, E. Sáez, J.C. del Amo, J. Am. Chem. Soc. 120 (1998) 812;

(p) A.C. Albéniz, P. Espinet, R. Manrique, A. Pérez-Mateo, Angew. Chem. Int. Ed. 41 (2002) 2363;

(q) J. Barluenga, L.A. López, O. Löber, M. Tomás, S. García-Granda, C. ÀlvarezRúa, J. Borge, Angew. Chem. Int. Ed. 40 (2001) 3392;

(r) J. Barluenga, P. Barrio, R. Vicente, L.A. López, M. Tomás, J. Organomet. Chem. 689 (2004) 3793;

(s) E.O. Fischer, M. Böck, J. Organomet. Chem. 287 (1985) 279;

(t) M. Fañanás-Mastral, F. Aznar, Organometallics 28 (2009) 666

[12] (a) I.J.B. Lin, C.S. Vasam, Coord. Chem. Rev. 251 (2007) 642;

(b) J.C. Garrison, W.J. Youngs, Chem. Rev. 105 (2005) 3978 (and references therein).

[13] (a) L.R. Titcomb, S. Caddick, F.G.N. Cloke, D.J. Wilson, D. McKerrecher, Chem. Commun. (2001) 1388;

(b) F. Ozawa, M. Fujimori, T. Yamamoto, A. Yamamoto, Organometallics 5 (1986) 2144;

(c) F. Ozawa, K. Kurihara, M. Fujimori, T. Hidaka, T. Toyoshima, A. Yamamoto, Organometallics 8 (1989) 180;

(d) A.L. Casado, J.A. Casares, P. Espinet, Organometallics 16 (1997) 5730.

[14] (a) A.A.D. Tulloch, A.A. Danopoulos, S. Winston, S. Kleinhenz, G. Eastham, J. Chem. Soc. Dalton Trans. (2000) 4499;

(b) C.-Y. Wang, Y.-H. Liu, S.-M. Peng, S.-T. Liu, J. Organomet. Chem. 691 (2006) 401.

[15] H.M.J. Wang, I.J.B. Lin, Organometallics 17 (1998) 972.

[16] H.M. Peng, G. Song, Y. Li, X. Li, Inorg. Chem. 47 (2008) 8031.

[17] Notably, in the case of complexes bearing di-isopropyl-phenyl and mesityl groups as substituents of the imidazole $(\mathbf{3 b}, \mathbf{3 c}$ and $\mathbf{3 e}, \mathbf{3 f})$ free rotation about 
the $\mathrm{C}-\mathrm{N}$ bond is hindered and the splitting of signals related to the ortho groups of the aromatic ring is observed

[18] S. Filipuzzi, P.S. Pregosin, A. Albinati, S. Rizzato, Organometallics 27 (2008) 437

[19] L. Canovese, F. Visentin, C. Levi, A. Dolmella, Dalton Trans. 40 (2011) 966.

[20] L. Canovese, F. Visentin, C. Levi, C., Santo, V. Bertolasi, Inorg. Chim. Acta 390 (2012) 105

[21] F. Visentin, A. Togni, Organometallics 26 (2007) 3746

[22] (a) B. Crociani, S. Antonaroli, G. Bandoli, L. Canovese, F. Visentin, P. Uguagliati, Organometallics 18 (1999) 1137;

(b) L. Canovese, F. Visentin, C. Santo, G. Chessa, V. Bertolasi, Organometallics 29 (2010) 3027;

(c) L. Canovese, F. Visentin, C. Levi, C. Santo, V. Bertolasi, Inorg. Chim. Acta 378 (2011) 239.

[23] The reaction between silver(I) carbene derivatives and NHC palladium( 0 ) olefin complexes is reversible despite the precipitation of $\mathrm{AgBr}$ (see Ref. [20]).

[24] A.T. Normand, A. Stasch, L.-L. Ooi, K.J. Cavell, Organometallics 27 (2008) 6507.
[25] C.-F. Fu, C.-C. Lee, Y.-H. Liu, S.-M. Peng, S. Warsink, C.J. Elsevier, J.-T. Chen, S.-T. Liu, Inorg. Chem. 49 (2010) 3011.

[26] M.N. Burnett, C.K. Johnson, ORTEP III, Report ORNL-6895, Oak Ridge National Laboratory, Oak Ridge, TN, 1996.

[27] F.R. Hartley, S.R. Jones, J. Organomet. Chem. 66 (1974) 472.

[28] P.R. Auburn, P.B. Mackenzie, B. Bosnich, J. Am. Chem. Soc. 107 (1985) 2033.

[29] Z. Otwinowski, W. Minor, in: C.W. Carter, R.M. Sweet (Eds.), Methods in Enzymology, vol. 276, Academic Press, London, 1997, p. 307 (Part A).

[30] R.H. Blessing, Acta Crystallogr. Sect. A 51 (1995) 33.

[31] A. Altomare, M.C. Burla, M. Camalli, G.L. Cascarano, C. Giacovazzo, A. Guagliardi, A.G. Moliterni, G. Polidori, R. Spagna, J. Appl. Crystallogr. 32 (1999) 115.

[32] G.M. Sheldrick, SHELX-97, Program for Crystal Structure Refinement, University of Gottingen, Germany, 1997.

[33] M. Nardelli, J. Appl. Crystallogr. 28 (1995) 659.

[34] L.J. Farrugia, J. Appl. Crystallogr. 32 (1999) 837. 